\title{
MULTIPLE SOLUTIONS OF PERTURBED SUPERQUADRATIC SECOND ORDER HAMILTONIAN SYSTEMS
}

\author{
YIMING LONG
}

\begin{abstract}
In this paper we prove the existence of infinitely many distinct $T$ periodic solutions for the perturbed second order Hamiltonian system $\ddot{q}+$ $V^{\prime}(q)=f(t)$ under the conditions that $V: \mathbf{R}^{N} \rightarrow \mathbf{R}$ is continuously differentiable and superquadratic, and that $f$ is square integrable and $T$-periodic. In the proof we use the minimax method of the calculus of variation combining with a priori estimates on minimax values of the corresponding functionals.
\end{abstract}

\section{INTRODUCTION AND MAIN RESULTS}

Recently multiple existence results of variational problems which are invariant under a group of symmetries have been studied extensively. For forced vibration problems this kind of symmetry breaks down. Our paper is devoted to such perturbation problems for superquadratic second order Hamiltonian systems

$$
\ddot{q}+V^{\prime}(q)=f(t) .
$$

Our main result is the following

Theorem 1.2. Assume $V$ satisfies

(V1) $V \in C^{1}\left(\mathbf{R}^{N}, \mathbf{R}\right)$,

(V2) there are constants $\mu>2, r_{0}>0$ such that

$$
0<\mu V(q) \leq V^{\prime}(q) \cdot q \text { for }|q| \geq r_{0} .
$$

Then for any given $T, R>0$ and $T$-periodic function $f \in L^{2}\left([0, T], \mathbf{R}^{N}\right)$, the Hamiltonian system (1.1) possesses a $T$-periodic solution $q(t)$ with

$$
\max _{t \in[0, T]}|q(t)| \geq R \text {. }
$$

Here $\ddot{q}=d^{2} q / d t^{2}, V^{\prime}$ is the gradient of $V$, and we denote by $p \cdot q$ the scalar product in $\mathbf{R}^{N}$.

Received by the editors July 8, 1987 and, in revised form, December 10, 1987.

1980 Mathematics Subject Classification (1985 Revision). Primary 34C25, 58E05.

Key words and phrases. Classical Hamiltonian system, perturbation, minimax method, $S^{1}$ action, a priori estimates.

Sponsored in part by the U.S. Air Force under Grant No. AFOSR-87-0202. Reproduction in whole or in part is permitted for any purpose of the United States Government. 
For the autonomous case of (1.1) (i.e. $f \equiv 0$ ), the result was first proved by Rabinowitz [15, 16]. His proof is based on that the corresponding functional, $I(q)=\int_{0}^{T}\left(\frac{1}{2}|\dot{q}|^{2}-V(q(t))\right) d t$, is invariant under an action of group $S^{1}$. That is $I(q)=I\left(q_{\theta}\right)$ for any $\theta \in[0, T]$, where $q_{\theta}(t)=q(t+\theta)$. But in (1.1) the forcing term $f(t)$ destroys the group symmetry. In [4], Bahri and Berestycki used a Morse theory type argument and finite dimensional approximation to treat the system (1.1). They proved the above result by assuming $\left(\mathrm{V} 1^{\prime}\right): V \in$ $C^{2}\left(\mathbf{R}^{N}, \mathbf{R}\right)$ and (V2). In [18] Rabinowitz also considered such perturbation problems and established a functional framework, which works more directly on infinite dimensional spaces. He proved this result by assuming (V1), (V2) and some polynomial growth condition on $V$.

In this paper we follow the basic functional framework of [18], but we modify the treatment of the $S^{1}$-action and derive some new a priori estimates. These allow us to get Theorem 1.2. More precisely we prove Theorem 1.2 via the following steps.

$1^{\circ}$. In $\S \S 2$ and 3 , we define a modified functional $J$ corresponding to (1.1) and an auxiliary space $X$ with a simpler $S^{1}$-action on it than on $W^{1,2}\left(S^{1}, \mathbf{R}^{N}\right)$. With the aid of $X$ we define two sequences of minimax values, $\left\{b_{k, i}\right\}$ and $\left\{a_{k, i}\right\}$ for $k \in \mathbf{N}, i=1, \ldots, N$, of $J$, which satisfy $b_{k, i} \geq a_{k, i}$. We prove that if $b_{k, i}>a_{k, i}$ then $J$ possesses a critical value not less than $b_{k, i}$ and corresponding critical points of $J$ are solutions of (1.1).

$2^{\circ}$. In $\S 4$ we prove that if $b_{k, i}=a_{k, i}$ for all large enough $k$ and $i=$ $1, \ldots, N$, then this implies that

$$
a_{k, i} \leq \alpha k^{\mu /(\mu-1)}
$$

for some $\alpha>0$, large enough $k$ and $i=1, \ldots, N$. The proof depends on the properties of the $S^{1}$-action we defined on $X$.

$3^{\circ}$. In $\S 5$ we prove a lower estimate for the growth of $\left\{a_{k, i}\right\}$

$$
\lim _{k \rightarrow \infty} \frac{a_{k, i}}{k^{2}}=+\infty, \quad i=1, \ldots, N .
$$

(1.4) improves the corresponding estimate obtained in [18]. In (1.4), the exponent " 2 " is crucial, since if $\mu>2,(1.4)$ contradicts (1.3) and completes the proof of Theorem 1.2. The method we used to prove (1.4) is different from earlier known methods (for example [4, 18]).

In $\S 6$, we briefly describe some further extensions.

In the appendix we make a detailed study of a single ODE to get estimates used in $\S 5$.

This paper is a part of my doctoral thesis. I wish to express my sincere thanks to my advisor Professor Paul H. Rabinowitz for his guidance, help and encouragement.

\section{A MODIFIED FUNCTIONAL}

In order to prove the main Theorem 1.2, firstly, without loss of generality, we may assume $T=2 \pi$, so $f \in L^{2}\left(S^{1}, \mathbf{R}^{N}\right)$. Define $E=W^{1,2}\left(S^{1}, \mathbf{R}^{N}\right)$ with 
the norm

$$
\|q\|_{E}=\left(\int_{0}^{2 \pi}\left(|\dot{q}|^{2}+|q|^{2}\right) d t\right)^{1 / 2} \text { for } q \in E .
$$

We consider the following functional on $E$,

$$
I(q)=\int_{0}^{2 \pi}\left(\frac{1}{2}|\dot{q}|^{2}-V(q)+f \cdot q\right) d t .
$$

By standard results [20], we know that $I \in C^{1}(E, \mathbf{R})$ and the critical points of $I$ in $E$ will be weak solutions of (1.1). We define an $S^{1}$-action $T_{\theta}$ on $E$ by

$$
\left(T_{\theta} q\right)(t)=q(t+\theta) \text { for } \theta \in[0,2 \pi] .
$$

We say a functional $L: E \rightarrow \mathbf{R}$ is $S^{1}(E)$-invariant if

$$
L\left(T_{\theta} q\right)=L(q), \quad \forall q \in E, \theta \in[0,2 \pi] .
$$

If $f \equiv 0$ in (1.1), then the corresponding functional $I$ is $S^{1}(E)$-invariant. This important property was used by Rabinowitz (cf . [15, 16]) and others (cf. $[6,17])$ to prove the existence of multiple solutions of the autonomous problem. But when $f$ does depend on $t$, these proofs break down. In order to measure and control the asymmetry caused by $f(t)$, in [18], Rabinowitz introduced the following modified functional $J$. For the proofs of its properties we refer to [18].

It is easy to check that (V2) implies that there are constants $K_{1}, K_{2}, K_{3}>0$, such that

$$
\frac{1}{\mu}\left(V^{\prime}(q) \cdot q+K_{1}\right) \geq V(q)+K_{2} \geq K_{3}|q|^{\mu} \quad \forall q \in \mathbf{R}^{N} .
$$

Lemma 2.4 (Lemma 2.6 [18]). If $q$ is a critical point of $I$, then there exists $a$ constant $K_{4}>0$ depending on $\|f\|_{L^{2}}$ such that

$$
\int_{0}^{2 \pi}\left(V(q)+K_{2}\right) d t \leq \frac{1}{\mu} \int_{0}^{2 \pi}\left(V^{\prime}(q) \cdot q+K_{1}\right) d t \leq K_{4}\left(I^{2}(q)+1\right)^{1 / 2} \text {. }
$$

Let $\chi \in C^{\infty}(\mathbf{R}, \mathbf{R})$ such that

$$
\chi(t)=\left\{\begin{array}{ll}
1, & t \leq 1, \\
0, & t \geq 2,
\end{array} \text { and }-2<\chi^{\prime}<0 \text { for } t \in(1,2)\right.
$$

Let $\psi(q)=\chi\left(\left(2 K_{4}\left(I^{2}(q)+1\right)^{1 / 2}\right)^{-1} \int_{0}^{2 \pi}\left(V(q)+K_{2}\right) d t\right)$. Define for $q \in E$

$$
J(q)=\int_{0}^{2 \pi}\left(\frac{1}{2}|\dot{q}|^{2}-V(q)+\psi(q) f \cdot q\right) d t .
$$

Let $\operatorname{supp} \psi$ denote the closure of the set $\{q \in E \mid \psi(q) \neq 0\}$ in $E$.

Lemma 2.6 (Lemma $2.11[18]$ ). There is a constant $K_{5}>0$ depending on $\|f\|_{L \text { : }}$ such that

$$
\left|J(q)-J\left(T_{\theta} q\right)\right| \leq K_{5}\left(|J(q)|^{1 / \mu}+1\right)
$$

for all $q \in E$ and $\theta \in[0,2 \pi]$. 
Lemma 2.8 (Lemma 2.14 [18]). $J \in C^{1}(E, \mathbf{R})$ and there is a constant $K_{6}>0$ such that $J(q) \geq K_{6}$ and $J^{\prime}(q)=0$ implies that $J(q)=I(q)$ and $I^{\prime}(q)=0$.

For a given Banach space $\mathscr{B}$, we say a functional $L \in C^{1}(\mathscr{B}, \mathbf{R})$ satisfies the Palais-Smale condition (P.S) if whenever a sequence $\left\{u_{m}\right\} \subset \mathscr{B}$ satisfies that $L\left(u_{m}\right)$ is uniformly bounded and $L^{\prime}\left(u_{m}\right) \rightarrow 0$, then $\left\{u_{m}\right\}$ is precompact.

Let $[J]^{c}=\{q \in E \mid J(q) \leq c\},[J]_{c}=\{q \in E \mid J(q) \geq c\}$ for any $c \in \mathbf{R}$.

Lemma 2.9 (Lemma $2.15[18]$ ). There is a constant $K_{7}>0$ such that $J$ satisfies (P.S) on $[J]_{K_{7}}$.

\section{A MINIMAX FRAMEWORK}

In this section using the properties of the functional $J$ we define two sequences of minimax values of $J,\left\{b_{k, i}\right\}$ and $\left\{a_{k, i}\right\}$, such that $b_{k, i} \geq a_{k, i}$. We prove that whenever $b_{k, i}>a_{k, i}, J$ possesses a critical value not less than $b_{k, i}$ and corresponding critical points of $J$ are solutions of (1.1).

To define $\left\{b_{k, i}\right\}$ and $\left\{a_{k, i}\right\}$, we introduce an auxiliary space $X$ with an $S^{1}$-action $\hat{T}_{\theta}$. This structure will be used in $\S 4$ to get the estimates from above for the growth of $\left\{a_{k, i}\right\}$. The definitions of $b_{k, i}$ and $a_{k, i}$ involve the FadellRabinowitz cohomological index which will be used in $\S 5$ to get the estimates from below for the growth of $\left\{a_{k, i}\right\}$.

We define the usual lexicographical order for 2-tuples $(k, i) \in \mathscr{D}$ as following, where $\mathscr{D}=(\{0\} \cup \mathbf{N}) \times\{1, \ldots, N\}$.

$$
\begin{array}{ll}
(j, m)=(k, i), & \text { if } j=k \text { and } m=i, \\
(j, m)<(k, i), & \text { if } j<k \text { or } j=k \text { and } m<i .
\end{array}
$$

For convenience, we write $(k, i) \equiv\left(k+\left[\frac{i}{N}\right], i-\left[\frac{i}{N}\right] N\right)$ for any $i \in \mathbf{N}$, where $[a]$ is the integer part of $a,(k, 0) \equiv(k-1, N)$ for $k \in \mathbf{N}$.

Let $\xi_{m}, m=1, \ldots, N$, denote the usual orthonormal basis in $\mathbf{R}^{N}$. Define

$$
v_{j, m}=(\sin j t) \xi_{m}, \quad w_{j, m}=(\cos j t) \xi_{m} \quad \text { for }(j, m) \in \mathscr{D} .
$$

These functions form an orthogonal basis for $E$. Let

$$
E_{k, i}=\operatorname{span}\left\{v_{j, m}, w_{j, m} \mid(0,1) \leq(j, m) \leq(k, i)\right\} \quad \text { for }(k, i) \in \mathscr{D} .
$$

Let $R_{0}>0$ be a constant, which will be determined later. By $(2.5)$, there is a constant $R_{k, i} \geq R_{0}+1$ for any $(k, i) \in \mathscr{D}$ such that

$$
J(q) \leq 0 \text { if } q \in E_{k, i} \text { and }\|q\|_{E} \geq R_{k, i},
$$

and

$$
R_{k, i+1}>R_{k, i}>k \quad \forall(k, i) \in \mathscr{D} .
$$

We shall impose more conditions on $R_{k, i}$ 's later. Define

$$
D_{k, i}(E)=B_{k, i}(E) \cap E_{k, i},
$$

where $B_{k, i}(E)=B_{R_{k, i}}(E), B_{\rho}(E)=\left\{q \in E \mid\|q\|_{E} \leq \rho\right\}$ for $\rho>0$. 
For the $S^{1}$-action $T_{\theta}$ defined on $E$ by (2.1), we say a subset $B$ of $E$ is $S^{1}(E)$-invariant if

$$
T_{\theta} q \in B \quad \forall q \in B, \theta \in[0,2 \pi] .
$$

If $B$ is an $S^{1}(E)$-invariant set of $E$, we say $h: B \rightarrow E$ is an $S^{1}(E)$-equivariant map, if

$$
h\left(T_{\theta} q\right)=T_{\theta} h(q) \quad \forall q \in B, \theta \in[0,2 \pi] .
$$

Note that the fixed point set of this group of symmetries is

$$
\operatorname{Fix}\left\{T_{\theta}\right\} \equiv\left\{q \in E \mid T_{\theta} q=q \forall \theta \in[0,2 \pi]\right\}=E_{0, N} .
$$

Let $\mathscr{E}$ denote the family of closed (in $E$ ) invariant subsets of $E \backslash\{0\}$.

In order to analyse the effect of the $S^{1}$-action $T_{\theta}$ on $E$, for $q \in E$, we write its Fourier expansion in the following polar coordinate form,

$$
q=\sum_{m=1}^{N} \rho_{0, m} w_{0, m}+\sum_{(j, m) \geq(1,1)} \rho_{j, m}\left(\cos \varphi_{j, m} v_{j, m}+\sin \varphi_{j, m} w_{j, m}\right)
$$

where $\rho_{0, m} \in \mathbf{R}$ for $m=1, \ldots, N, \rho_{j, m} \geq 0, \varphi_{j, m} \in[0,2 \pi)$ for $(1,1) \leq$ $(j, m)$. If $\rho_{j, m}=0$ then $\varphi_{j, m}=0$. We also write $q=\left(\rho_{j, m}, \varphi_{j, m}\right)$ with $\varphi_{0, m} \equiv 0$ for $m=1, \ldots, N$. Then a computation shows that for $\theta \in \mathbf{R}$,

$$
\begin{aligned}
T_{\theta} q= & \sum_{m=1}^{N} \rho_{0, m} w_{0, m} \\
& +\sum_{(j, m) \geq(1,1)} \rho_{j, m}\left\{\cos \left(\varphi_{j, m}+j \theta\right) v_{j, m}+\sin \left(\varphi_{j, m}+j \theta\right) w_{j, m}\right\}
\end{aligned}
$$

Using the expression (3.4), for $q \in E$, we find

$$
\|q\|_{E}^{2}=\int_{0}^{2 \pi}\left(|\dot{q}|^{2}+|q|^{2}\right) d t=\sum_{m=1}^{N}\left(2 \pi \rho_{0, m}^{2}+\pi \sum_{j \geq 1}\left(1+j^{2}\right) \rho_{j, m}^{2}\right) .
$$

In order to define a family of the minimax sets, we introduce a new space $X$ based on $E$. We shall define a simpler $S^{1}$-action on $X$ than that on $E$.

$$
X=\mathbf{R}^{N} \times \prod_{(j, m) \geq(1,1)} \mathbf{C}_{j, m}, \quad \text { where } \mathbf{C}_{j, m} \equiv \mathbf{C} .
$$

For $x \in X$, we write

$$
x=\sum_{m=1}^{N} \rho_{0, m} \zeta_{0, m}+\sum_{(j, m) \geq(1,1)} \rho_{j, m} e^{i \varphi_{j, m}} \zeta_{j, m},
$$

where $i=\sqrt{-1}, \rho_{0, m} \in \mathbf{R}$ for $m=1, \ldots, N, \rho_{j, m} \geq 0, \varphi_{j, m} \in[0,2 \pi)$ and $\rho_{j, m}=0$ implies $\varphi_{j, m}=0$ for $(j, m) \geq(1,1) .\left\{\zeta_{j, m} \mid(j, m) \in \mathscr{D}\right\}$ is defined 
as following

$$
\zeta_{j, m}=\left(a_{0,1}, \ldots, a_{0, N} ; a_{1,1}, \ldots, a_{1, N} ; \ldots\right) \in \prod_{k=1}^{\infty} \prod_{n=1}^{N}, \ldots, R_{k, n},
$$

where $\mathbf{R}_{k, n} \equiv \mathbf{R}, a_{j, m}=1$ and $a_{k, n}=0$ if $(k, n) \neq(j, m)$. We also write $x=\left(\rho_{j, m}, \varphi_{j, m}\right)$.

The norm on $X$ is defined by

$$
\|x\|_{X}=\left(\sum_{m=1}^{N}\left(2 \pi \rho_{0, m}^{2}+\pi \sum_{j \geq 1}\left(1+j^{2}\right) \rho_{j, m}^{2}\right)\right)^{1 / 2},
$$

for $x \in X$ given by (3.7), and then $X$ becomes a Hilbert space under the corresponding inner products. We define an $S^{1}$-action $\hat{T}_{\theta}$ on $X$ by

$$
\hat{T}_{\theta} x=\sum_{m=1}^{N} \rho_{0, m} \zeta_{0, m}+\sum_{(j, m) \geq(1,1)} \rho_{j, m} e^{i\left(\varphi_{j, m}+\theta\right)} \zeta_{j, m},
$$

for $x \in X$ given by (3.7) and $\theta \in \mathbf{R}$. In a similar fashion to what was done above for $E$, we use (3.1), (3.2) and (2.4) to define $S^{1}(X)$-invariant subsets of $X, S^{1}(X)$-equivariant maps and $S^{1}(X)$-invariant functionals. Let $\mathscr{Z}$ denote the family of closed (in $X) S^{1}(X)$-invariant sets in $X \backslash\{0\}$. For $B \in \mathscr{Z}$, we say a map $h: B \rightarrow E$ is $S^{1}(X, E)$-equivariant if

$$
h\left(\hat{T}_{\theta} x\right)=T_{\theta} h(x) \quad \forall x \in B, \theta \in[0,2 \pi) .
$$

Let $X_{k, i}=\left\{x \in X \mid \rho_{j, m}(x)=0\right.$ if $\left.(k, i)<(j, m)\right\}$ for any $(k, i) \in \mathscr{D}$, i.e.

$$
X_{k, i}=\mathbf{R}^{N} \times \prod_{(j, m)=(1,1)}^{(k, i)} \mathbf{C}_{j, m}
$$

Note that $X_{0, N}=\operatorname{Fix}\left\{\hat{T}_{\theta}\right\}$.

On the Cartesian product space $X \times E$, we define an $S^{1}$-action by

$$
\tilde{T}_{\theta}(x, q)=\left(\hat{T}_{\theta} x, T_{\theta} q\right), \quad \text { for }(x, q) \in X \times E \text { and } \theta \in[0,2 \pi) .
$$

We define $S^{1}(X \times E)$-invariant sets, equivariant maps, and invariant functionals similarly. Let $\mathscr{F}$ denote the family of closed (in $X \times E) S^{1}(X \times E)$ )-invariant subsets in $(X \times E) \backslash\{0\}$. Then $\mathscr{F}$ contains sets in $\mathscr{X} \times\{0\}$ and $\{0\} \times \mathscr{E}$. We introduce the Fadell-Rabinowitz cohomological index theory (cf. [10]) on $\mathscr{F}$. Its properties we need are summarized in the following Lemma,

Lemma 3.10. There is an index theory on $\mathscr{F}$, i.e. a mapping $\tilde{\gamma}: \mathscr{F} \rightarrow\{0\} \cup \mathbf{N} \cup$ $\{\infty\}$ such that if $A, B \in \mathscr{F}$,

$1^{\circ} . \tilde{\gamma}(A) \leq \tilde{\gamma}(B)$, if there exists $h \in C(A, B)$ with $h$ being $S^{1}(X \times E)$ equivariant.

$2^{\circ} . \tilde{\gamma}(A \cup B) \leq \tilde{\gamma}(A)+\tilde{\gamma}(B)$. 
$3^{\circ}$. If $B \subset(X \times E) \backslash\left(X_{0, N} \times E_{0, N}\right)$ and $B$ is compact, then $\tilde{\gamma}(B)<\infty$, and there exists a constant $\delta>0$ such that $\tilde{\gamma}\left(\mathcal{N}_{\delta}(B, X \times E)\right)=\tilde{\gamma}(B)$, where $\mathscr{N}_{\delta}(B, X \times E)=\left\{z \in X \times E \mid\|z-B\|_{X \times E} \leq \delta\right\}$.

$4^{\circ}$. If $\mathscr{S} \subset(X \times E) \backslash\left(X_{0, N} \times E_{0, N}\right)$ is a $2 n-1$ dimensional invariant sphere, then $\tilde{\gamma}(\mathscr{S})=n$.

Define

$$
\hat{\gamma}=\left.\tilde{\gamma}\right|_{\mathscr{Z} \times\{0\}}, \quad \gamma=\left.\tilde{\gamma}\right|_{\{0\} \times \mathscr{E}} .
$$

They induce index theories $\hat{\gamma}, \gamma$ on $\mathscr{X}$ and $\mathscr{E}$ separately by identifying $\mathscr{X}$ with $\mathscr{Z} \times\{0\}$ and $\mathscr{E}$ with $\{0\} \times \mathscr{E}$. $\hat{\gamma}$ and $\gamma$ possess the properties listed in Lemma 3.10 too. Furthermore on the relationship between $\hat{\gamma}$ and $\gamma$, we have

Lemma 3.11. For $A \in \mathscr{X}, B \in \mathscr{E}$, if there exists $h \in C(A, B)$ with $h$ being $S^{1}(X, E)$-equivariant, then $\hat{\gamma}(A) \leq \gamma(B)$.

Proof. This is a direct consequence of $1^{\circ}$ of Lemma 3.10. Q.E.D.

Let $D_{k, i}(X)=B_{k, i}(X) \cap X_{k, i}$, where $B_{k, i}(X)=B_{R_{k, i}}(X)=\left\{x \in X \mid\|x\|_{X} \leq\right.$ $\left.R_{k, i}\right\}$. For $x \in X, q \in E$ we write $x \sim q$ if $\rho_{j, m}(x)=\rho_{j, m}(q)$ and $\varphi_{j, m}(x)=$ $\varphi_{j, m}(q)$ for any $(j, m) \in \mathscr{D}$.

The usual "identity map", $\operatorname{id}(x)=q$ if $x \sim q$, is not $S^{1}(X, E)$-equivariant. We need to define a new map which is $S^{1}(X, E)$-equivariant and which will play the role of the identity map in the $(X, E)$ setting. For any $x \in X$ with expression (3.7) we define a map $h$ as follows

$$
h(x)=\sum_{m=1}^{N} \rho_{0, m} w_{0, m}+\sum_{(j, m) \geq(1,1)} \rho_{j, m}\left(\cos \left(j \varphi_{j, m}\right) v_{j, m}+\sin \left(j \varphi_{j, m}\right) w_{j, m}\right) .
$$

Concerning this map $h$, we have

Lemma 3.13. The map $h$ defined by (3.12) possesses the following properties,

$1^{\circ} . h \in C(X, E)$.

$2^{\circ}$. $h$ is $S^{1}(X, E)$-equivariant.

$3^{\circ}$. $h\left(\partial B_{\rho}(X) \cap X_{k, i}\right)=\partial B_{\rho}(E) \cap E_{k, i}, \forall(k, i) \in \mathscr{D}$ and $\rho>0$.

$4^{\circ}$. If $q \sim x \in X_{0, N}, h(x)=q$.

Proof. $1^{\circ}$. By (3.6) and (3.8), $x \in X$ implies that $\|h(x)\|_{E}=\|x\|_{X}<\infty$, i.e. $h(x) \in E$. Hence $h$ maps $X$ into $E$. It is clear $h(X)=E$. We only prove the continuity of $h$ for the case $N=1$. The general case can be done similarly. Suppose $x_{n}, x \in X, x_{n} \rightarrow x$ in $X$ as $n \rightarrow \infty$. Write $x_{n}=\left(\rho_{n}(n), \varphi_{k}(n)\right)$, $x=\left(\rho_{k}, \varphi_{k}\right)$, where $\varphi_{0}(n) \equiv \varphi_{0} \equiv 0$. Then

$$
\begin{gathered}
2 \pi\left(\rho_{0}(n)-\rho_{0}\right)^{2}+\pi \sum_{k=1}^{\infty}\left(1+k^{2}\right)\left(\rho_{k}^{2}(n)+\rho_{k}^{2}-2 \rho_{k}(n) \rho_{k} \cos \left(\varphi_{k}(n)-\varphi_{k}\right)\right) \\
=\left\|x_{n}-x\right\|_{X}^{2} \rightarrow 0 \text { as } n \rightarrow \infty
\end{gathered}
$$


Since $\|x\|^{2}=2 \pi \rho_{0}^{2}+\pi \sum_{k=1}^{\infty}\left(1+k^{2}\right) \rho_{k}^{2}<\infty$, given any $\varepsilon>0$, there is an $N_{1} \in \mathbf{N}$ such that

$$
\pi \sum_{k=N_{1}}^{\infty}\left(1+k^{2}\right) \rho_{k}^{2}<\varepsilon
$$

Since

$$
\begin{aligned}
\pi \sum_{k=N_{\mathrm{l}}}^{\infty}\left(1+k^{2}\right) \rho_{k}^{2}(n) & \leq \pi \sum_{k=N_{1}}^{\infty}\left(1+k^{2}\right)\left(2 \rho_{k}^{2}+2\left(\rho_{k}(n)-\rho_{k}\right)^{2}\right) \\
& \leq 2 \pi \sum_{k=N_{\mathrm{l}}}^{\infty}\left(1+k^{2}\right) \rho_{k}^{2}+2\left\|x_{n}-x\right\|_{X}^{2}
\end{aligned}
$$

there is $N_{2} \in \mathbf{N}$ such that for any $n \geq N_{2}$

$$
\pi \sum_{k=N_{\mathrm{l}}}^{\infty}\left(1+k^{2}\right) \rho_{k}^{2}(n)<3 \varepsilon
$$

Since $x_{n} \rightarrow x$ in $X$ as $n \rightarrow \infty$, there is $N_{3} \in \mathbf{N}$ such that for $n \geq N_{3}$,

$$
\begin{aligned}
I_{1}(n) & \equiv 2 \pi\left(\rho_{0}(n)-\rho_{0}\right)^{2} \\
& +\pi \sum_{k=1}^{N_{1}-1}\left(1+k^{2}\right)\left(\rho_{k}^{2}(n)+\rho_{k}^{2}-2 \rho_{k}(n) \rho_{k} \cos k\left(\varphi_{k}(n)-\varphi_{k}\right)\right)<\varepsilon .
\end{aligned}
$$

Let $N_{4}=\max \left\{N_{2}, N_{3}\right\}$. From (3.14)-(3.17), we get that for any $n \geq N_{4}$,

$$
\begin{aligned}
& \left\|h\left(x_{n}\right)-h(x)\right\|_{E}^{2} \\
& \quad=I_{1}(n)+\pi \sum_{k=N_{1}}^{\infty}\left(1+k^{2}\right)\left(\rho_{k}^{2}(n)+\rho_{k}^{2}-2 \rho_{k}(n) \rho_{k} \cos k\left(\varphi_{k}(n)-\varphi_{k}\right)\right) \\
& \quad \leq I_{1}(n)+2 \pi \sum_{k=N_{1}}^{\infty}\left(1+k^{2}\right)\left(\rho_{k}^{2}(n)+\rho_{k}^{2}\right)<9 \varepsilon .
\end{aligned}
$$

Thus $h \in C(X, E)$.

$2^{\circ}$. For any $x=\left(\rho_{j, m}, \varphi_{j, m}\right) \in X, h(x)=\left(\rho_{j, m}, j \varphi_{j, m}\right) \in E$. Then for any $\theta \in \mathbf{R}$,

$$
\begin{aligned}
h\left(\hat{T}_{\theta} x\right) & =h\left(\left(\rho_{j, m}, \varphi_{j, m}+\theta\right)\right)=\left(\rho_{j, m}, j\left(\varphi_{j, m}+\theta\right)\right) \\
& =T_{\theta}\left(\rho_{j, m}, j \varphi_{j, m}\right)=T_{\theta} h(x) .
\end{aligned}
$$

Therefore $h$ is $S^{1}(X, E)$-equivariant on $X$.

$3^{\circ}$ and $4^{\circ}$ are direct consequences of the definition of $h$. This completes the proof. Q.E.D. 
Definition 3.18. We denote the above map $h$ by "id" : $X \rightarrow E$.

With the aid of the map id, we can now define a minimax framework. For $(k, i) \geq(1,1)$, define

$$
\begin{gathered}
\Gamma_{k, i}=\left\{h \in C\left(D_{k, i}(X), E\right) \mid h \text { is } S^{1}(X, E) \text {-equivariant on } D_{k, i}(X)\right. \text { and } \\
\left.h=\text { id on }\left(D_{k, i}(X) \cap X_{0, N}\right) \cup\left(\partial B_{k, i}(X) \cap X_{k, i}\right)\right\}, \\
\Lambda_{k, i}=\left\{h \in C\left(D_{k, i+1}(X), E\right)|h|_{D_{k, i}(X)} \in \Gamma_{k, i} \text { and } h=\right.\text { id on } \\
\left.\left(\partial B_{k, i+1}(X) \cap X_{k, i+1}\right) \cup\left(\left(B_{k, i+1}(X) \backslash B_{k, i}(X)\right) \cap X_{k, i}\right)\right\}, \\
\mathscr{A}_{k, i}=\left\{h \left(\overline{\left.D_{j, m}(X) \backslash Y\right) \mid(j, m) \geq(k, i), h \in \Gamma_{j, m} \text { and } Y \in \mathscr{X}}\right.\right. \\
\text { with } \hat{\gamma}(Y) \leq(j N+m)-(k N+i)\}, \\
\mathscr{B}_{k, i}=\left\{h \left(\overline{\left.D_{j, m+1}(X) \backslash Y\right)} \mid(j, m) \geq(k, i), h \in \Lambda_{j, m} \text { and } Y \in \mathscr{X}\right.\right. \\
\text { with } \hat{\gamma}(Y) \leq(j N+m)-(k N+i)\} .
\end{gathered}
$$

We define two sequences of minimax values of $J$ as follows

$$
a_{k, i}=\inf _{A \in \mathscr{A}_{k, i}} \max _{q \in A} J(q), \quad b_{k, i}=\inf _{B \in \mathscr{B}_{k, i}} \max _{q \in B} J(q) .
$$

Remark 3.19. $1^{\circ}$. Since id $\in \Gamma_{j, m} \cap \Lambda_{j, m}, \Gamma_{j, m} \neq \varnothing, \Lambda_{j, m} \neq \varnothing$ for any $(j, m) \geq(1,1)$.

$2^{\circ}$. In $\S 5$ we shall prove that $a_{k, i}, b_{k, i}$ are finite for $(k, i) \geq(1,1)$.

Lemma 3.20. $1^{\circ}$. $a_{k, i} \leq a_{k, i+1}$ for any $(k, i) \geq(1,1)$.

$2^{\circ}$. $a_{k, i} \leq b_{k, i}$ for any $(k, i) \geq(1,1)$.

Proof. $1^{\circ}$ follows since $\mathscr{A}_{k, i+1} \subset \mathscr{A}_{k, i}$ and $2^{\circ}$ holds since for any $B \in \mathscr{B}_{k, i}$ there is a set $A \subset B$ with $A \in \mathscr{A}_{k, i}$. Q.E.D.

In [18], Rabinowitz proved an important existence result (Lemmas 1.57 and 2.29 [18]), which shows that if $b_{k, i}>a_{k, i}$ then the asymmetric functional $J$ possesses a critical value $b_{k, i}(\delta) \geq b_{k, i}$. The following proposition is a variant of this result in our setting, which is one of the three key steps in the proof of Theorem 1.2.

Proposition 3.21. Let $(k, i) \geq(1,1)$. If $b_{k, i}>a_{k, i} \geq K_{7}$, let $\delta \in\left(0, b_{k, i}-a_{k, i}\right)$ and

$$
\mathscr{B}_{k, i}(\delta)=\left\{h \left(\overline{\left.D_{j, m+1}(X) \backslash Y\right)} \in \mathscr{B}_{k, i} \mid J(q) \leq a_{k, i}+\delta \text {, if } q \in h\left(\overline{\left.D_{j, m}(X) \backslash Y\right)}\right\},\right.\right.
$$
let

$$
b_{k, i}(\delta)=\inf _{B \in \mathscr{B}_{k, i}(\delta)} \max _{q \in B} J(q)
$$

Then $b_{k, i}(\delta)$ is a critical value of $J$.

Remark 3.22. $1^{\circ}$. Since for any $h \in \Gamma_{j, m}$, we can extend it to a map in $\Lambda_{j, m}$, $\mathscr{B}_{k, i}(\delta) \neq \varnothing$. In fact for $h \in \Gamma_{j, m}$, extending $h$ as id on

$$
\left(\partial B_{j, m+1}(X) \cap X_{j, m}+1\right) \cup\left(\left(B_{j, m+1}(X) \backslash B_{j, m}(X)\right) \cap X_{j, m}\right)
$$


and using the Dugundji Extension Theorem [8] to further extend $h$ to the whole $D_{j, m+1}(X)$, we get that $h \in \Lambda_{j, m}$.

$2^{\circ} . b_{k, i}(\delta) \geq b_{k, i}>a_{k, i} \geq K_{7}$.

For the proof of Proposition 3.21, we require the following standard "deformation theorem" (cf. [14]).

Lemma 3.23. Let $\dot{J} \in C^{1}(E, \mathbf{R})$ satisfy $(P . S)$ on $[J]_{a}$. Then if $c>a, \bar{\varepsilon}>0$ and $c$ is not a critical value of $J$, there exists $\varepsilon \in(0, \bar{\varepsilon})$ and $\eta \in C([0,1] \times E, E)$ such that

$$
\begin{aligned}
& 1^{\circ} \cdot \eta(t, q)=q \text { if } q \notin J^{-1}(c-\bar{\varepsilon}, c+\bar{\varepsilon}) . \\
& 2^{\circ} \cdot \eta\left(1,[J]^{c+\varepsilon}\right) \subseteq[J]^{c-\varepsilon} .
\end{aligned}
$$

Proof of Proposition 3.21. Choose $\bar{\varepsilon}=\frac{1}{2}\left(b_{k, i}-a_{k, i}-\delta\right)>0$. If $b_{k, i}(\delta)$ is not a critical value of $J$ then there exist $\varepsilon$ and $\eta$ as given by Lemma 3.23. Choose $B \in \mathscr{B}_{k, i}(\delta)$ such that

$$
\max _{q \in B} J(q) \leq b_{k, i}(\delta)+\varepsilon
$$

By the definition of $\mathscr{B}_{k, i}(\delta)$, there are $(j, m) \geq(k, i), H \in \Lambda_{j, m}$ and $Y \in \mathscr{X}$ with $\hat{\gamma}(Y) \leq(j N+m)-(k N+i)$ such that $B=H\left(\overline{D_{j, m+1}(X) \backslash Y}\right)$. Now we define a new map $h$ as following

$$
\begin{gathered}
h=\eta(1, H) \quad \text { on } \overline{D_{j, m+1}(X) \backslash Y}, \\
h=H \quad \text { on } B_{j, m+1}(X) \cap X_{j, m} \cap Y, \\
h=\mathrm{id} \quad \text { on } \partial B_{j, m+1}(X) \cap X_{j, m+1} .
\end{gathered}
$$

Let

$$
\begin{gathered}
Q=\left(\overline{\left.D_{j, m+1}(X) \backslash Y\right)} \cup\left(B_{j, m+1}(X) \cap X_{j, m} \cap Y\right) \cup\left(\partial B_{j, m+1}(X) \cap X_{j, m+1}\right),\right. \\
P=\left(D_{j, m+1}(X) \cap Y\right) \backslash X_{j, m} .
\end{gathered}
$$

For any $x \in \overline{D_{j, m}(X) \backslash Y}$, since $B \in \mathscr{B}_{k, i}(\delta)$,

$$
J(H(x)) \leq a_{k, i}+\delta \leq b_{k, i}-2 \bar{\varepsilon}<b_{k, i}(\delta)-\bar{\varepsilon},
$$

so by $1^{\circ}$ of Lemma $3.23, \eta(1, H(x))=H(x)$.

For any $x$ in

$$
\left(\partial B_{j, m+1}(X) \cap X_{j, m+1}\right) \cup\left(\left(B_{j, m+1}(X) \backslash B_{j, m}(X)\right) \cap X_{j, m}\right),
$$

since $H \in \Lambda_{j, m}, H(x)=\mathrm{id}(x)$, so

$$
J(H(x))=J(\operatorname{id}(x)) \leq 0<b_{k, i}(\delta)-\bar{\varepsilon} .
$$

Thus $\eta(1, H(x))=H(x)$ by $1^{\circ}$ of Lemma 3.23 .

The above arguments show that the definitions (3.25), (3.26) and (3.27) are consistent and $h \in C(Q, E)$. From the above proof, we also get that

$$
h=H \quad \text { on }\left(B_{j, m+1}(X) \cap X_{j, m}\right) \cup\left(\partial B_{j, m+1}(X) \cap X_{j, m+1}\right) .
$$


Since $\partial P \subseteq Q=\bar{Q}$, where " $\partial$ " is taken within $X_{j, m+1}$, by the Dugundji Extension Theorem [8] we can extend $h$ to all of $P$ continuously. This shows that $h \in C\left(D_{j, m+1}(X), E\right)$. By (3.29) and that $H \in \Lambda_{j, m}, h \in \Lambda_{j, m}$, and thus $B_{1} \equiv h\left(\overline{\left.D_{j, m+1}(X) \backslash Y\right)} \in \mathscr{B}_{k, i}\right.$. By (3.28), $B_{1} \in \mathscr{B}_{k, i}(\delta)$.

Now (3.24), (3.25) and $2^{\circ}$ of Lemma 3.23 show that

$$
\max _{q \in B_{1}} J(q) \leq b_{k, i}(\delta)-\varepsilon .
$$

But (3.30) contradicts the definition of $b_{k, i}(\delta)$.

The proof is complete. Q.E.D.

\section{AN UPPER ESTIMATE FOR THE GROWTH OF $\left\{a_{k, i}\right\}$}

If the system (1.1) possesses only finitely many solutions, for at most finitely many $(k, i), b_{k, i}>a_{k, i}$. In this section we prove that if for all large enough $k$ and $i=1, \ldots, N, b_{k, i}=a_{k, i}$, then using the inequality (2.7) we have

$$
a_{k, i+1} \leq a_{k, i}+K_{5}\left(a_{k, i}^{1 / \mu}+1\right) \text {, }
$$

and this inequality implies the following estimate on the growth of $\left\{a_{k, i}\right\}$

$$
a_{k, i} \leq \beta_{1} k^{\mu /(\mu-1)} \text {. }
$$

By (2.7), we get that

$$
J\left(T_{\theta} q\right) \leq J(q)+K_{5}\left(|J(q)|^{1 / \mu}+1\right) \quad \forall q \in E, \theta \in[0,2 \pi] .
$$

Since $\mu>1$, there is $K_{8}>0$ depending on $K_{5}$ and $\mu$ only such that

$$
J(q)+K_{5}\left(|J(q)|^{1 / \mu}+1\right) \leq 0 \text { if } J(q) \leq-K_{8} .
$$

We shall prove the following claim in $\S 5$,

$$
a_{k, i} \rightarrow+\infty \text { as } k \rightarrow+\infty \text { for any } 1 \leq i \leq N .
$$

So there is a $k_{0} \in \mathbf{N}$ such that

$$
a_{k, i} \geq K_{8} \quad \forall(k, i) \geq\left(k_{0}, 1\right) .
$$

Proposition 4.5. Assume that there is a constant $k_{1} \geq k_{0}$ such that $b_{k, i}=a_{k, i}$ for every $k \geq k_{1}$ and $1 \leq i \leq N$. Then there exists a constant $\beta_{1}=\beta_{1}\left(k_{1}\right)>0$ such that

$$
a_{k, i} \leq \beta_{1} k^{\mu /(\mu-1)}, \quad \text { for } k \geq k_{1}, 1 \leq i \leq N .
$$

Proof. For $(k, i) \geq\left(k_{1}, 1\right)$, we get that

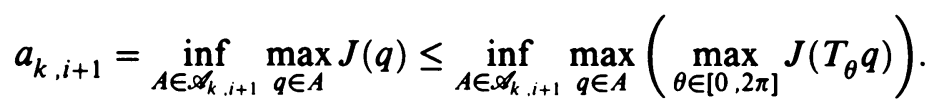

Assuming the following inequality for a moment

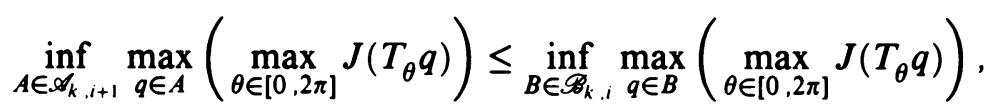


we get that

$$
a_{k, i+1} \leq \inf _{B \in \mathscr{B}_{k, i}} \max _{q \in B}\left(\max _{\theta \in[0,2 \pi]} J\left(T_{\theta} q\right)\right)
$$

For any $\varepsilon>0$, by the definition of $b_{k, i}$, there is a $B \in \mathscr{B}_{k, i}$ such that

$$
\max _{q \in B} J(q) \leq b_{k, i}+\varepsilon=a_{k, i}+\varepsilon .
$$

For this choice of $B$, using (4.1), (4.2), (4.4), and (4.10), we get

$$
J\left(T_{\theta} q\right) \leq a_{k, i}+\varepsilon+K_{5}\left(\left(a_{k, i}+\varepsilon\right)^{1 / \mu}+1\right) \quad \forall q \in B, \theta \in[0,2 \pi] .
$$

(4.9) and (4.11) yield

$$
a_{k, i+1} \leq a_{k, i}+\varepsilon+K_{5}\left(\left(a_{k, i}+\varepsilon\right)^{1 / \mu}+1\right) .
$$

Let $\varepsilon \rightarrow 0$, we get that

$$
a_{k, i+1} \leq a_{k, i}+K_{5}\left(a_{k, i}^{1 / \mu}+1\right) .
$$

Then a slight extension of the argument of [2] gives (4.6).

Thus we have reduced the proof of (4.6) to proving the inequality (4.8), i.e. the following lemma.

Lemma 4.12. If $L$ is a continuous $S^{1}(E)$-invariant functional on $E$, then

$$
\inf _{A \in \mathscr{A}_{k, i+1}} \max _{q \in A} L(q) \leq \inf _{B \in \mathscr{B}_{k, i}} \max _{q \in B} L(q)
$$

is true for $(k, i) \geq(1,1)$.

Proof. It suffices to prove that for every $B \in \mathscr{B}_{k, i}$, there is an $A \in \mathscr{A}_{k, i+1}$ such that

$$
\max _{q \in A} L(q) \leq \max _{q \in B} L(q) .
$$

Given $B \in \mathscr{B}_{k, i}$, by the definition of $\mathscr{B}_{k, i}$, there is $(j, m) \geq(k, i), H \in \Lambda_{j, m}$ and $Y \in \mathscr{X}$ with $\hat{\gamma}(Y) \leq(j N+m)-(k N+i)$, such that $B=H\left(\overline{D_{j, m+1}(X) \backslash Y}\right)$. Let

$$
\begin{aligned}
U_{j, m}(X)=\left\{x \in D_{j, m+1}(X) \mid x=x^{\prime}+\rho_{j, m+1} \zeta_{j, m+1},\right. & x^{\prime} \in X_{j, m}, \\
\rho_{j, m+1} & \left.\geq 0 \text { and }\|x\|_{X} \leq R_{j, m+1}\right\} .
\end{aligned}
$$

Now we define a map $h$ from which we shall get an $A \in \mathscr{A}_{k, i+1}$. Let

$$
\begin{gathered}
h(x)=H(x) \text { for } x \in U_{j, m}(X), \\
h\left(\hat{T}_{\theta} x\right)=T_{\theta} h(x) \text { for } x \in U_{j, m}(X) \text { and } \theta \in[0,2 \pi) .
\end{gathered}
$$

We need to show that $h \in \Gamma_{j, m+1}$. Since for any $y \in D_{j, m+1}(X)$, there exists only one $x \in U_{j, m}(X)$ and only one $\theta \in[0,2 \pi)$ such that

$$
y=\hat{T}_{\theta} x,
$$


$h$ is well defined on $D_{j, m+1}(X)$. Since $H \in \Lambda_{j, m}, h=H \in C\left(U_{j, m}(X), E\right)$ and $h=H$ is $S^{1}(X, E)$-equivariant on $D_{j, m+1}(X) \cap X_{j, m}$. The $S^{1}$ action is continuous and (4.16) does not change the values of $h$ on $D_{j, m+1}(X) \cap X_{j, m}$. Thus $h \in C\left(D_{j, m+1}(X), E\right)$. By the above argument and the definition (4.15), (4.16), we have that $h$ is $S^{1}(X, E)$-equivariant on $D_{j, m+1}(X)$ and $h=H=$ id on $\left(D_{j, m+1}(X) \cap X_{0, N}\right) \cup\left(\partial B_{j, m+1}(X) \cap X_{j, m+1}\right)$. Therefore $h \in \Gamma_{j, m+1}$. Let $A=h\left(\overline{D_{j, m+1}(X) \backslash Y}\right)$, then $A \in \mathscr{A}_{k, i+1}$. For any $y \in \overline{D_{j, m+1}(X) \backslash Y}$, there exists $x \in \overline{U_{j, m}(X) \backslash Y}$ and $\theta \in[0,2 \pi)$ such that $\hat{T}_{\theta} x=y$. So

$$
h(y)=h\left(\hat{T}_{\theta} x\right)=T_{\theta} h(x)=T_{\theta} H(x)
$$

Since

$$
\overline{U_{j, m}(X) \backslash Y} \subseteq \overline{D_{j, m+1}(X) \backslash Y},
$$

we get that

$$
L(h(y))=L\left(T_{\theta} H(x)\right)=L(H(x)) \leq \max _{q \in B} L(q) .
$$

Thus

$$
\max _{q \in A} L(q) \leq \max _{q \in B} L(q) .
$$

The proof is complete. Q.E.D.

Remark 4.18. A result like Proposition 4.5 was given by Rabinowitz (Lemma 2.31 [18]). Unfortunately the proof there is not complete. By introducing the space $X$ we get a unique expression $y=\hat{T}_{\theta} x$ in (4.17), which guarantees the map $h$ constructed in the proof is well defined, and enables us to complete the proof. We are indebted to Professor Paul H. Rabinowitz who pointed out the shortcoming in the proof of Lemma 2.31 [18] to us.

\section{A LOWER ESTIMATE FOR THE GROWTH OF $\left\{a_{k, i}\right\}$}

In this section, we shall prove the following estimate on the minimax value sequence $\left\{a_{k, i}\right\}$ of $J$.

Proposition 5.1.

$$
\lim _{k \rightarrow+\infty} \frac{a_{k, i}}{k^{2}}=+\infty, \quad \text { for } i=1,2, \ldots, N .
$$

At the end of this section, we shall complete the proof of our main result, Theorem 1.2.

We will prove Proposition 5.1 in several steps. These steps reduce the estimates to successively simpler situations, the final one being an estimate of minimax values for functional associated with a single ordinary differential equation.

Step 1. Reducing to the estimate to a sequence of minimax values $\left\{c_{k, i}^{*}\right\}$ for a functional $\Phi(q)=\sum_{i=1}^{N} \varphi\left(q_{i}\right)$.

We need the following lemma, which was proved by Bahri and Berestycki, 
Lemma 5.3 (Lemma 5.1 [4]). Let $U \in C\left(\mathbf{R} \times \mathbf{R}^{N}, \mathbf{R}\right)$ and $U(t, q)$ is $T$-periodic in $t$, then there exists $G \in C^{2}(\mathbf{R}, \mathbf{R})$ such that

$1^{\circ} . G^{\prime}=g$ is odd.

$2^{\circ}$. $G(0)=g(0)=g^{\prime}(0)=0$.

$3^{\circ} . g$ is increasing and convex on $[0,+\infty)$.

$4^{\circ} .0<3 G(r) \leq r g(r)$ for all $r \in \mathbf{R} \backslash\{0\}$.

$5^{\circ} . \quad U(t, q) \leq \sum_{i=1}^{N} G\left(q_{i}\right)+U_{0}$ for all $(t, q) \in \mathbf{R} \times \mathbf{R}^{N}$, where $q=$ $\left(q_{1}, \ldots, q_{N}\right)$ and the constant $U_{0}=1+\max _{|q| \leq 1,0 \leq t \leq T}|U(t, q)|$.

Proof. For completeness, we sketch the proof here. For $n \in \mathbf{N}$, set $m_{n}=$ $\max _{|q| \leq n, 0 \leq t \leq T} U(t, q)$. Choose a sequence of positive numbers $a_{0}, a_{1}, \ldots$, $a_{n}, \ldots$, such that $\sum_{i=0}^{n} a_{i} \geq m_{n+1}$. For $r \geq 0$, define

$$
g(r)=3 \sqrt{N} \sum_{n=1}^{\infty} a_{n}\left((\sqrt{N} r-n+1)^{+}\right)^{2},
$$

where $C^{+}=\max (0, C)$, and $G(r)=\int_{0}^{r} g(s) d s$. For $r<0$, define $g(r)=$ $-g(-r)$, and $G(r)=G(-r)$. This $G$ does the job. Q.E.D.

We define a Sobolev space $W=W^{1,2}\left(S^{1}, \mathbf{R}\right)$ with norm

$$
\|u\|_{W}=\left(\int_{0}^{2 \pi}\left(|\dot{u}|^{2}+|u|^{2}\right) d t\right)^{1 / 2} \quad \forall u \in W .
$$

Then $E=W^{N}$ (a Cartesian product of spaces). In Lemma 5.3, let $U \equiv$ $V(q)+\frac{1}{2}|q|^{2}$ for $q \in \mathbf{R}^{N}$, let $T=2 \pi$, to define $G$ and $g$. Then we define

$$
\phi(u)=\int_{0}^{2 \pi}\left(\frac{1}{2}|\dot{u}|^{2}-G(u)\right) d t \quad \forall u \in W,
$$

and

$$
\Phi(q)=\int_{0}^{2 \pi}\left(\frac{1}{2}|\dot{q}|^{2}-\sum_{i=1}^{N} G\left(q_{i}\right)\right) d t=\sum_{i=1}^{N} \phi\left(q_{i}\right), \quad \text { for } q=\left(q_{1}, \ldots, q_{N}\right) \in E .
$$

We have the following standard result.

Lemma 5.5. $\phi \in C^{2}(W, \mathbf{R}), \Phi \in C^{2}(E, \mathbf{R})$, and both satisfy the corresponding Palais-Smale condition (P.S).

Remark. Cf. the proof of Theorem 2.61 of [15] and [20].

We require one more condition on the $R_{k, i}$ 's,

$$
\Phi(q) \leq 0 \quad \text { if } q \in E_{k, i} \text { and }\|q\|_{E} \geq R_{k, i} .
$$

This is possible by $4^{\circ}$ of Lemma 5.3 and inequalities similar to (2.3) for $G$.

We define for $(k, i) \geq(1,1)$

$$
c_{k, i}^{*}=\inf _{A \in \mathscr{P}_{k}, i} \max _{q \in A} \Phi(q) .
$$

We shall show that $c_{k, i}^{*}>-\infty$. Firstly we have the following lemma: 
Lemma 5.7. For $(k, i) \geq(1,1)$

$$
c_{k, i}^{*} \leq c_{k, i+1}^{*}
$$

and

$$
a_{k, i} \geq c_{k, i}^{*}-2 \pi C_{1}
$$

where $C_{1}=\frac{1}{2}\|f\|_{L^{2}}^{2}+2 \pi\left(1+\left.\max _{|q| \leq 1}\left|V(q)+\frac{1}{2}\right| q\right|^{2} \mid\right)$.

Proof. The first inequality follows from that $\mathscr{A}_{k, i+1} \subseteq \mathscr{A}_{k, i}$. Since

$$
\begin{aligned}
J(q) & =\int_{0}^{2 \pi}\left(\frac{1}{2}|\dot{q}|^{2}-V(q)+\psi(q) f \cdot q\right) d t \\
& \geq \int_{0}^{2 \pi}\left(\frac{1}{2}|\dot{q}|^{2}-V(q)\right) d t-\|f\|_{L^{2}}\|q\|_{L^{2}} \\
& \geq \int_{0}^{2 \pi}\left(\frac{1}{2}|\dot{q}|^{2}-\left(V(q)+\frac{1}{2}|q|^{2}\right)\right) d t-\frac{1}{2}\|f\|_{L^{2}}^{2} .
\end{aligned}
$$

Therefore by the choice of $G$, for $q \in E$ we have that

$$
J(q) \geq \int_{0}^{2 \pi}\left(\frac{1}{2}|\dot{q}|^{2}-\sum_{i=1}^{N} G\left(q_{i}\right)\right) d t-2 \pi C_{1}=\Phi(q)-2 \pi C_{1} .
$$

This yields (5.8). Q.E.D.

Step 2. Reducing to the estimate to a sequence of minimax values $\left\{c_{k, i}\right\}$ for $\phi$.

In [19], Rabinowitz proved the following intersection result, which is essential for the lower bound estimate of the $a_{k, i}$ 's.

Lemma 5.9 (Proposition 1.19 and Corollary 1.25 [19]). If $(j, m) \geq(k, i) \geq$ $(1,1), h \in \Lambda_{j, m}, Y \in \mathscr{X}$ with $\hat{\gamma}(Y) \leq(j N+m)-(k N+i)$, and $0<\rho<R_{j, m}$, then

$$
h\left(\overline{D_{j, m}(X) \backslash Y}\right) \cap \partial B_{\rho}(E) \cap\left(E_{k, i-1}\right)^{\perp} \neq \varnothing .
$$

Remark. Note that $h\left(\partial B_{j, m}(X) \cap X_{j, m}\right)=\partial B_{j, m}(E) \cap E_{j, m}$ plays the role of the identity map from $\partial B_{j, m}(E) \cap E_{j, m}$ onto itself in the proof of Proposition 1.19 [19]. The proof there used an $S^{1}$-action version of the Borsuk-Ulam theorem proved by Fadell, Husseini and Rabinowitz [9]. We refer the readers to [19] and [9], and omit the proof here.

In $W$, we define subspaces

$$
W_{k}=\operatorname{span}\{\sin j t, \cos j t \mid 0 \leq j \leq k\} \quad \text { for } k \in\{0\} \cup \mathbf{N} .
$$

Then

$$
E_{k, i-1} \supseteq E_{k-1, N} \supseteq W_{k-1}^{N}
$$

Thus

$$
\left(E_{k, i-1}\right)^{\perp} \subseteq\left(W_{k-1}^{N}\right)^{\perp}=\left(W_{k-1}^{\perp}\right)^{N} \text {. }
$$


So we can rewrite (5.10) as follows

$$
h\left(\overline{D_{j, m}(X) \backslash Y}\right) \cap \partial B_{\rho}(E) \cap\left(W_{k-1}^{\perp}\right)^{N} \neq \varnothing .
$$

For elements in $\left(W_{k-1}^{\perp}\right)^{N}$, we have the following results

Lemma 5.12. For $k>1, q=\left(q_{1}, \ldots, q_{N}\right) \in\left(W_{k-1}^{\perp}\right)^{N}$, we have that

$$
k\|q\|_{L^{2}} \leq\|\dot{q}\|_{L^{2}},
$$

and

$$
\left\|q_{i}\right\|_{L^{\infty}} \leq\left(\frac{2}{\pi(k-1)}\right)^{1 / 2}\left\|\dot{q}_{i}\right\|_{L^{2}}^{2} \quad \forall i=1, \ldots, N .
$$

Proof. $1^{\circ}$. If $q \in\left(W_{k-1}^{\perp}\right)^{N}$, then by (3.5),

$$
q=\sum_{(j, m) \geq(k, 1)} \rho_{j, m}\left(\cos \varphi_{j, m} v_{j, m}+\sin \varphi_{j, m} w_{j, m}\right)
$$

where $\rho_{j, m} \geq 0$ and $0 \leq \varphi_{j, m}<2 \pi, \rho_{j, m}=0$ implies $\varphi_{j, m}=0$. Therefore a computation shows that

$$
\begin{aligned}
\|\dot{q}\|_{L^{2}}^{2} & =\pi \sum_{(j, m) \geq(k, 1)} j^{2} \rho_{j, m}^{2} \\
& \geq k^{2} \pi \sum_{(j, m) \geq(k, 1)} \rho_{j, m}^{2}=k^{2}\|q\|_{L^{2}}^{2} .
\end{aligned}
$$

This is (5.13).

$2^{\circ}$. By the same computation as in (5.15), we get that for every $i=$ $1, \ldots, N$

$$
\left\|\dot{q}_{i}\right\|_{L^{2}}^{2}=\pi \sum_{j \geq k} j^{2} \rho_{j, i}^{2}
$$

and

$$
\begin{aligned}
\left\|q_{i}\right\|_{L^{\infty}} & \leq \sqrt{2} \sum_{j \geq k}\left(j \rho_{j, i}\right) \frac{1}{j} \leq \sqrt{2}\left(\sum_{j \geq k} j^{2} \rho_{j, i}^{2}\right)^{1 / 2}\left(\sum_{j \geq k} \frac{1}{j^{2}}\right)^{1 / 2} \\
& \leq\left(\frac{2}{\pi(k-1)}\right)^{1 / 2}\left\|\dot{q}_{i}\right\|_{L^{2}}
\end{aligned}
$$

(by (5.16)). This yields (5.14) and completes the proof. Q.E.D.

Using the intersection Lemma 5.9 and estimates (5.13) and (5.14), we can get a positive lower bound for $c_{k, i}^{*}$ with large enough $(k, i)$.

Lemma 5.17. Fix $R_{0}=4$. Then there is $k_{1}>1$ such that

$1^{\circ}$. For any $(k, i) \geq\left(k_{1}, 1\right)$ and $A \in \mathscr{A}_{k, i}$, there exists $q \in A$ such that

$$
\Phi(q) \geq 2 \text { and } \phi\left(q_{n}\right) \geq-\frac{2}{N} \quad \forall n=1, \ldots, N .
$$


$2^{\circ}$. For $(k, i) \geq\left(k_{1}, 1\right)$

$$
c_{k, i}^{*} \geq 2
$$

Proof. $1^{\circ}$. For $A \in \mathscr{A}_{k, i}$, there are $(j, m) \geq(k, i), h \in \Gamma_{j, m}, Y \in \mathscr{X}$ with $\hat{\gamma}(Y) \leq(j N+m)-(k N+i)$ such that

$$
A=h\left(\overline{D_{j, m}(X) \backslash Y}\right) \text {. }
$$

By Lemma 5.9 and (5.11), there exists $x \in \overline{D_{j, m}(X) \backslash Y}$ such that

$$
q \equiv h(x) \in \partial B_{R_{0}}(E) \cap\left(W_{k-1}^{\perp}\right)^{N},
$$

so

By (5.13)

$$
q_{n} \in W_{k-1}^{\perp} \text { for } n=1, \ldots, N, \quad q \equiv\left(q_{1}, \ldots, q_{N}\right) .
$$

Thus

$$
R_{0}^{2}=\|q\|_{E}^{2} \leq\left(1+\frac{1}{k^{2}}\right)\|\dot{q}\|_{L^{2}}^{2} .
$$

$$
R_{0}^{2} \geq\|\dot{q}\|_{L^{2}}^{2} \geq \frac{1}{2} R_{0}^{2} \text { and }\left\|\dot{q}_{n}\right\|_{L^{2}} \leq R_{0} \text { for } n=1, \ldots, N .
$$

Fix $R_{0}=4$. Then there is $k_{1}>1$ such that if $k \geq k_{1}$

$$
C_{2} \equiv \max _{|s| \leq 4 \sqrt{2 /(\pi(k-1))}} G(s) \leq \frac{1}{N \pi} .
$$

If $(k, i) \geq\left(k_{1}, 1\right)$, we have that

$$
\Phi(q)=\int_{0}^{2 \pi}\left(\frac{1}{2}|\dot{q}|^{2}-\sum_{n=1}^{N} G\left(q_{n}\right)\right) d t \geq 4-2 N \pi C_{2} \geq 2 .
$$

For $n=1, \ldots, N$, by (5.20) and (5.21) we get that

$$
\phi\left(q_{n}\right)=\int_{0}^{2 \pi}\left(\frac{1}{2}\left|\dot{q}_{n}\right|^{2}-G\left(q_{n}\right)\right) d t \geq-\int_{0}^{2 \pi} G\left(q_{n}\right) d t \geq-\frac{2}{N} .
$$

This proves $(5.18)$.

$2^{\circ}$. Denote the above $q$ of $A$ by $q_{A}$. From $1^{\circ}$ we get that

$$
\max _{q \in A} \Phi(q) \geq \Phi\left(q_{A}\right) \geq 2 \quad \forall A \in \mathscr{A}_{k, i}
$$

Thus $c_{k, i}^{*} \geq 2$ if $(k, i) \geq\left(k_{1}, 1\right)$. This completes the proof. Q.E.D.

Remark 5.22. In the above proof, if we let $R_{0}=\sqrt{\pi(k-1) / 2}$, then for $(k, i) \geq$ $(2,1)$ it yields that

$$
\begin{aligned}
\Phi(q) & \geq \frac{1}{2} \cdot \frac{k^{2}}{k^{2}+1} \cdot \frac{\pi}{2}(k-1)-2 N \pi \max _{|s| \leq 1} G(s) \\
& \geq \frac{\pi}{5}(k-1)-2 N \pi \max _{|s| \leq 1} G(s),
\end{aligned}
$$


and

$$
c_{k, i}^{*} \geq \frac{\pi}{5}(k-1)-2 N \pi \max _{|s| \leq 1} G(s)
$$

For the case $k=1,(5.14)$ becomes

$$
\left\|q_{i}\right\|_{L^{\infty}} \leq \sqrt{\frac{\pi}{3}}\left\|\dot{q}_{i}\right\|_{L^{2}} \quad \text { for every } i=1, \ldots, N \text {. }
$$

Let $R_{0}=\sqrt{\pi / 3}$. Then a similar computation proves that

$$
c_{1, i}^{*} \geq \frac{3}{4 \pi}-2 N \pi \max _{|s| \leq 1} G(s) \quad \text { for every } i=1, \ldots, N \text {. }
$$

Therefore for $(k, i) \geq(1,1), c_{k, i}^{*}, a_{k, i}, b_{k, i}$ are all finite.

(5.23) also shows that $c_{k, i}^{*}$ grows at least linearly in $k$ as $k \rightarrow+\infty$. This implies (4.3). But we need a much stronger estimate, i.e.

$$
\lim _{k \rightarrow+\infty} \frac{c_{k, i}^{*}}{k^{2}}=+\infty \text { for } i=1, \ldots, N
$$

Now we can define a sequence of minimax values of $\phi$. For $(k, i) \geq\left(k_{1}, 1\right)$, where $k_{1}$ is given by Lemma 5.17 , and $A \in \mathscr{A}_{k, i}$, we define

$$
\begin{aligned}
& S(A)=\left\{q_{n} \in W \mid \text { there exists } q=\left(q_{1}, \ldots, q_{n}, \ldots q_{N}\right) \in A\right. \text { such that } \\
& \left.\qquad \Phi(q) \geq 1, \phi\left(q_{n}\right) \geq \frac{1}{N} \text { and } \phi\left(q_{j}\right) \geq-2 \text { for } j=1, \ldots, N\right\} .
\end{aligned}
$$

Define

$$
c_{k, i}=\inf _{A \in \mathscr{A}_{k, i}} \max _{q_{n} \in S(A)} \phi\left(q_{n}\right) \text { for }(k, i) \geq\left(k_{1}, 1\right) .
$$

Then we have the following result on $c_{k, i}$ 's,

Lemma 5.24. For $(k, i) \geq\left(k_{1}, 1\right)$ :

$1^{\circ}$. $A \in \mathscr{A}_{k, i}$ implies $S(A) \neq \varnothing$.

$2^{\circ} . c_{k, i}^{*} \geq c_{k, i}-2(N-1)$.

$3^{\circ} . c_{k, i} \geq \frac{1}{N}$.

Proof. $1^{\circ}$. Since $(k, i) \geq\left(k_{1}, 1\right)$, by Lemma 5.17 , for any $A \in \mathscr{A}_{k, i}$, there is $q \in A$ such that

$$
\Phi(q) \geq 1 \text { and } \phi\left(q_{j}\right) \geq-2 \text { for } j=1, \ldots, N,
$$

and this implies that there is an $n \in\{1, \ldots, N\}$ such that $\phi\left(q_{n}\right) \geq \frac{1}{N}$, i.e. $q_{n} \in S(A)$. So $S(A) \neq \varnothing$.

$2^{\circ}$ is a direct consequence of the definitions of $S(A)$ and $c_{k, i}$.

$3^{\circ}$. For every $A \in \mathscr{A}_{k, i}$

$$
\max _{q_{n} \in S(A)} \phi\left(q_{n}\right) \geq \frac{1}{N}
$$

Thus $c_{k, i} \geq \frac{1}{i}$. Q.E.D. 
Step 3. The properties of critical values of $\phi$.

Bahri and Berestycki proved the following result about the critical values and critical points of $\phi$ on $W$. Consider the ordinary differential equation corresponding to $\phi$,

$$
\ddot{v}+g(v)=0 \text { for } v(t) \in \mathbf{R} .
$$

Lemma 5.27 (Propositions 4.1 and $4.2[4]$ ). $1^{\circ}$. The nontrivial critical values of $\phi$ on $W$ form a sequence $\left\{\gamma_{k}\right\}$, which possesses the following properties

$$
0<\gamma_{1}<\gamma_{2}<\cdots<\gamma_{k}<\gamma_{k+1}<\ldots, \quad \lim _{k \rightarrow+\infty} \frac{\gamma_{k}}{k^{2}}=+\infty .
$$

$2^{\circ}$. There exists a sequence of nontrivial $2 \pi$-periodic solutions $\left\{u_{k}\right\}$ of $(5.26)$ such that $u_{k}(0)=u_{k}(2 \pi)=0, u_{k}$ has exactly $2 k-1$ zeros (all are simple) in $(0,2 \pi)$ for $k \in \mathbf{N}$, and for $k \in \mathbf{N}$

$$
\mathscr{K}_{\gamma_{k}} \equiv\left(\phi^{\prime}\right)^{-1}(0) \cap \phi^{-1}\left(q_{k}\right)=\left\{T_{\theta} u_{k} \mid \theta \in[0,2 \pi]\right\} .
$$

Remark. In the appendix we give a more direct proof of Lemma 5.27 via phase plane analysis.

Step 4. The properties of $\left\{c_{k, i}\right\}$.

Since we can identify the space $W$ with the subspace $W \times\{0\}^{N-1}$ of $E$, we get the induced $S^{1}$-action $T_{\theta}$ on $W$. We define $S^{1}(W)$-invariant set, $S^{1}(W)$ equivariant map and $S^{1}(W)$-invariant functional in the same way as in (3.1), (3.2) and (2.2), and $\operatorname{Fix}\left\{\left.T_{\theta}\right|_{W}\right\} \equiv\left\{u \in W \mid T_{\theta} u=u \forall \theta \in[0,2 \pi]\right\}=W_{0}$. Let $\mathscr{W}$ be the family of closed (in $W$ ) $S^{1}(W)$-invariant sets in $W \backslash\{0\}$. From the index theory $\gamma: \mathscr{E} \rightarrow \overline{\mathbf{N}} \cup\{\infty\}$ we also get an induced index theory on $\mathscr{W}$. We still denote it by $\gamma$. It possesses the four properties listed in Lemma 3.10.

In order to study the properties of $\left\{c_{k, i}\right\}$, we need the following deformation lemma for $\phi$ and $\Phi$. We are rather sketchy here. For details of the proof we refer to Theorem 1.9 [14].

Lemma 5.28. Let $\phi \in C^{2}(W, \mathbf{R})$, be $S^{1}(W)$-invariant and satisfy $(P . S)$ on $W$. For $c \in \mathbf{R}$, let $\mathscr{K}_{c}=\left(\phi^{\prime}\right)^{-1}(0) \cap \phi^{-1}(c)$. Suppose $\mathscr{N}$ is any neighborhood of $\mathscr{K}_{c}$ in $W$. Let $\Phi(q)=\sum_{i=1}^{N} \phi\left(q_{i}\right)$, for $q=\left(q_{1}, \ldots, q_{N}\right) \in W^{N} \equiv E$. Then there exists $\eta \in C([0,1] \times E, E)$ and a constant $\bar{\varepsilon}>0$ such that for $\varepsilon \in(0, \bar{\varepsilon})$,

$1^{\circ} . \eta(0, q)=q$ for $q \in E$.

$2^{\circ}$. $\eta(t, \cdot)$ is $S^{1}(E)$-equivariant.

$3^{\circ} . \phi\left(\eta_{i}(t, q)\right) \leq \phi\left(\eta_{i}(s, q)\right)$ for $q \in E, 0 \leq s \leq t \leq 1$ and $i=1, \ldots, N$.

$4^{\circ}$. $\Phi(\eta(t, q)) \leq \Phi(\eta(s, q))$, for $q \in E, 0 \leq s \leq t \leq 1$.

$5^{\circ}$. If $\Phi(q) \leq 0, \eta(t, q)=q$ for every $t \in[0,1]$.

$6^{\circ}$. Let $q=\left(q_{1}, \ldots, q_{N}\right) \in E, \Phi(\eta(t, q)) \geq 1$ for every $t \in[0,1]$ and $\mathscr{K}_{c}=\varnothing$. Then for any $i \in\{1, \ldots, N\}$, if $q_{i} \in[\phi]^{c+\varepsilon}, \eta_{i}(1, q) \in[\phi]^{c-\varepsilon}$. 
$7^{\circ}$. More generally, let $q=\left(q_{1}, \ldots, q_{N}\right) \in E, \Phi(\eta(t, q)) \geq 1$ for every $t \in[0,1]$. Then for any $i \in\{1, \ldots, N\}$, if $q_{i} \in[\phi]^{c+\varepsilon} \backslash \mathcal{N}, \eta_{i}(1, q) \in[\phi]^{c-\varepsilon}$. Here $\eta(1, q)=\left(\eta_{1}(1, q), \ldots, \eta_{N}(1, q)\right)$.

Proof. Assume $\mathscr{K}_{c} \neq \varnothing$. If $\mathscr{K}_{c}=\varnothing$, the proof is simpler. By (P.S) for $\phi, \mathscr{K}_{c}$ is compact. So there is $\delta>0$ such that $M_{\delta} \equiv \operatorname{int} \mathscr{N}_{\delta}\left(\mathscr{K}_{c}, W\right) \subset \mathscr{N}$. Thus it suffices to prove $7^{\circ}$ with $\mathscr{N}$ replaced by $M_{\delta}$.

There are $b, \bar{\varepsilon}>0$ depending on $\delta$ such that

$$
\left\|\phi^{\prime}(u)\right\|_{W} \geq b \quad \forall u \in[\phi]^{c+\bar{\varepsilon}} \backslash\left([\phi]^{c-\bar{\varepsilon}} \cup M_{\delta / 8}\right) .
$$

Since (5.29) remains valid if $\bar{\varepsilon}$ decreases, we can take $\bar{\varepsilon}$ such that

$$
0<\bar{\varepsilon}<\min \left\{\frac{b \delta}{16}, \frac{b^{2}}{2}, 1\right\} .
$$

Let $\varepsilon \in(0, \bar{\varepsilon})$. Let $A=\{u \in W \mid \phi(u) \geq c+\bar{\varepsilon}$ or $\phi(u) \leq c-\bar{\varepsilon}\}$ and $B=\{u \in W \mid c-\varepsilon \leq \phi(u) \leq c+' \varepsilon\}$. Thus $A \cap B=\varnothing$. Define

$$
g_{1}(u)=\|u-A\|_{W}\left(\|u-A\|_{W}+\|u-B\|_{W}\right)^{-1} \quad \forall u \in W .
$$

Then $g_{1}$ is Lipschitz continuous with $g_{1}=0$ on $A, g_{1}=1$ on $B$ and $0 \leq g_{1}(u) \leq 1$. Similarly there is a Lipschitz continuous $g_{2}: W \rightarrow \mathbf{R}$ with $g_{2}=0$ on $M_{\delta / 8}, g_{2}=1$ on $W \backslash M_{\delta / 4}$ and $0 \leq g_{2}(u) \leq 1$. Since $\phi$ is $S^{1}(W)$ invariant, $g_{1}$ and $g_{2}$ can be taken to be $S^{1}(W)$-invariant. Define

$$
g_{3}(s)= \begin{cases}1 & \text { if } 0 \leq s \leq 1 \\ \frac{1}{s} & \text { if } 1 \leq s\end{cases}
$$

Then $g_{3}$ is Lipschitz continuous. Choose $g_{4} \in C^{\infty}(\mathbf{R}, \mathbf{R})$ such that $g_{4}(s)=0$ if $s \leq 0, g_{4}(s)=1$ if $s \geq 1$, and $0 \leq g_{4}(s) \leq 1$ for $0 \leq s \leq 1$. For $u \in W$, define

$$
\omega(u)=-g_{1}(u) g_{2}(u) g_{3}\left(\left\|\phi^{\prime}(u)\right\|_{W}\right) \phi^{\prime}(u) .
$$

For $q=\left(q_{1}, \ldots, q_{N}\right) \in W^{N} \equiv E$, define

$$
\boldsymbol{\Omega}(q)=g_{4}(\Phi(q))\left(\omega\left(q_{1}\right), \ldots, \omega\left(q_{N}\right)\right) .
$$

Then $\omega$ and $\Omega$ are $S^{1}$-equivariant, locally Lipschitz continuous vector fields on $W$ and $E$ respectively, and we have

$$
\begin{aligned}
& 0 \leq\|\omega(u)\|_{W} \leq 1 \quad \text { for } u \in W, \\
& 0 \leq\|\Omega(q)\|_{E} \leq \sqrt{N} \text { for } q \in E .
\end{aligned}
$$

We consider the following ordinary differential equation on $E$

$$
\left\{\begin{array}{l}
d \eta(t, q) / d t=\Omega(\eta(t, q)) \\
\eta(0, q)=q
\end{array} \quad \text { for } q \in E\right.
$$

By the basic existence theory for such equations and the properties of $\Omega$ described above, we obtain the existence of $\eta(t, q)$ on $(-\infty,+\infty) \times E$, and in particular we have $\eta(t, q) \in C([0,1] \times E, E)$. 
From (5.30) we get $1^{\circ}$ to $5^{\circ}$ easily. Under the condition $\Phi(\eta(t, q)) \geq 1$ for every $t \in[0,1]$, by the definition of $g_{4},(5.30)$ yields

$$
\left\{\begin{array}{l}
d \eta_{i}(t, q) / d t=\omega\left(\eta_{i}(t, q)\right), \\
\eta_{i}(0, q)=q_{i} .
\end{array}\right.
$$

Viewing $\left(q_{1}, \ldots, q_{i-1}, q_{i+1}, \ldots, q_{N}\right)$ as parameters in (5.31), using the definition of $\bar{\varepsilon}$, and following the proof in [14], we can get $6^{\circ}$ and $7^{\circ}$.

Therefore we have completed the proof of Lemma 5.28. Q.E.D.

With the aid of the above deformation lemma, we get the following multiplicity result for $\left\{c_{k, i}\right\}$,

Lemma 5.32. For $(k, i) \geq\left(k_{1}, 1\right)$ :

$1^{\circ} \cdot c_{k, i} \leq c_{k, i+1}$.

$2^{\circ} . c_{k, i}$ is a critical value of $\phi$.

$3^{\circ}$. Any critical point of $\phi$ corresponding to $c_{k, i}$ lies in $W \backslash W_{0}$.

$4^{\circ}$. If $c_{k, i+1}=\cdots=c_{k, i+l} \equiv c$ and $\mathscr{K} \equiv\left(\phi^{\prime}\right)^{-1}(0) \cap \phi^{-1}(c)$, then $\gamma(\mathscr{K}) \geq$ $\left[\frac{l-1}{N}\right]+1$.

Proof. $1^{\circ}$ holds since $\mathscr{A}_{k, i+1} \subseteq \mathscr{A}_{k, i}$. By $3^{\circ}$ of Lemma 5.28, $c_{k, i} \geq \frac{1}{N}$, and therefore $\mathscr{K}_{k, i} \cap W_{0}=\varnothing$. So $3^{\circ}$ holds. To prove $2^{\circ}$, it suffices to prove the stronger multiplicity assertion $4^{\circ}$.

Since $\phi$ satisfies (P.S) on $W, \mathscr{K}$ is compact. By $3^{\circ}, \mathscr{K} \subset W \backslash W_{0}$. By Lemma 3.10 for $\mathscr{W}$, there is $\delta>0$ such that $\gamma\left(M_{\delta}\right)=\gamma(\mathscr{K})$, where $M_{\delta}=$ $\mathscr{N}_{\delta}(\mathscr{K}, W)$. Let $\mathscr{N}=M_{\delta / 2}$, then by Lemma 5.28 , we get a deformation flow $\eta \in C([0,1] \times E, E)$ and a constant $\varepsilon>0$, which possess properties $1^{\circ}-7^{\circ}$ of Lemma 5.28. Assume $\gamma(\mathscr{K}) \leq\left[\frac{l-1}{N}\right]$. Choose $A \in \mathscr{A}_{k, i+l}$ such that

$$
\max _{q_{n} \in S(A)} \phi\left(q_{n}\right) \leq c+\varepsilon .
$$

Then by the definition of $\mathscr{A}_{k, i+l}$, there are $(j, m) \geq(k, i+l), h \in \Gamma_{j, m}$, $Y \in \mathscr{X}$ with $\hat{\gamma}(Y) \leq(j N+m)-(k N+i+l)$ such that

$$
A=h\left(\overline{D_{j, m}(X) \backslash Y}\right) \text {. }
$$

Let $Z=\bigcup_{p=1}^{N} h_{p}^{-1}\left(M_{\delta}\right)$, where $h=\left(h_{1}, \ldots, h_{N}\right): X \rightarrow E=W^{N}$. Then by Lemmas 3.10 and 3.11 , we get that

$$
\begin{aligned}
\hat{\gamma}(Z) & \leq \sum_{p=1}^{N} \hat{\gamma}\left(h_{p}^{-1}\left(M_{\delta}\right)\right) \leq \sum_{p=1}^{N} \gamma\left(M_{\delta}\right)=N \gamma(\mathscr{K}) \\
& \leq N\left[\frac{l-1}{N}\right] \leq l-1 .
\end{aligned}
$$

Let $B=h\left(\overline{D_{j, m}(X) \backslash(Y \cup Z)}\right)$. Since by Lemma 3.10

$$
\begin{aligned}
\hat{\gamma}(Y \cup Z) & \leq \hat{\gamma}(Y)+\hat{\gamma}(Z) \\
& \leq(j N+m)-(k N+i+l)+(l-1)=(j N+m)-(k N+i+1) .
\end{aligned}
$$

So $B \in \mathscr{A}_{k, i+1}$. 
Since $B \subseteq A, S(B) \subseteq S(A)$, so by $(5.33)$

$$
\max _{q_{n} \in S(B)} \phi\left(q_{n}\right) \leq c+\varepsilon
$$

We define

$$
H(x)=\eta(1, h(x)) \quad \text { for every } x \in D_{j, m}(X) .
$$

Then $H \in C\left(D_{j, m}(X), E\right)$, since $h$ and $\eta(1, \cdot)$ are continuous. $H$ is $S^{1}(X, E)$ equivariant, since so is $h$ and $\eta(1, \cdot)$ is $S^{1}(E)$-equivariant.

For $x \in \partial B_{j, m}(X) \cap X_{j, m}$, since $h \in \Gamma_{j, m}, h(x) \in \partial B_{j, m}(E) \cap E_{j, m}$ and

$$
\Phi(h(x)) \leq 0 \text {. }
$$

For $q \sim x \in D_{j, m}(X) \cap X_{0, N}$, since $h \in \Gamma_{j, m}, h(x)=q$ and

$$
\Phi(h(x))=\Phi(q) \leq 0 .
$$

Therefore in both cases by $5^{\circ}$ of Lemma 5.28, $H(x) \equiv \eta(1, h(x))=h(x)=$ $\operatorname{id}(x)$. This shows that $H \equiv \eta(1, h) \in \Gamma_{j, m}$. Therefore

$$
Q \equiv H\left(\overline{D_{j, m}(X) \backslash(Y \cup Z)}\right) \in \mathscr{A}_{k, i+1} .
$$

By the definition of $S(Q)$, for any $q_{n} \in S(Q)$, there exists

$$
x \in \overline{D_{j, m}(X) \backslash(Y \cup Z)}
$$

such that $q \equiv\left(q_{1}, \ldots, q_{n}, \ldots, q_{N}\right)=\eta(1, h(x))$. From the definition of $S(Q)$ and $3^{\circ}, 4^{\circ}$ of Lemma 5.28, we get that

(5.35) $1 \leq \Phi(q)=\Phi(\eta(1, h(x))) \leq \Phi(\eta(t, h(x))) \leq \Phi(\eta(0, h(x)))=\Phi(h(x))$ for every $t \in[0,1]$,

$$
\begin{gathered}
\frac{1}{N} \leq \phi\left(q_{n}\right)=\phi\left(\eta_{n}(1, h(x))\right) \leq \phi\left(\eta_{n}(0, h(x))\right)=\phi\left(h_{n}(x)\right), \\
-2 \leq \phi\left(q_{p}\right)=\phi\left(\eta_{p}(1, h(x))\right) \leq \phi\left(\eta_{p}(0, h(x))\right)=\phi\left(h_{p}(x)\right)
\end{gathered}
$$

for $p=1, \ldots, N$. Thus $h_{n}(x) \in S(B)$. By (5.34)

$$
\phi\left(h_{n}(x)\right) \leq c+\varepsilon .
$$

Since $x \notin Z=\bigcup_{p=1}^{N} h_{p}^{-1}\left(M_{\delta}\right), h_{n}(x) \notin \mathscr{N} \equiv M_{\delta / 2}$. Therefore

$$
h_{n}(x) \in[\phi]^{c+\varepsilon} \backslash \mathscr{N} \text {. }
$$

Now (5.35), (5.36) and $7^{\circ}$ of Lemma 5.28 imply that

$$
q_{n} \equiv \eta_{n}(1, h(x)) \in[\phi]^{c-\varepsilon} \text {. }
$$

Since $q_{n}$ is arbitrarily chosen from $S(Q)$, this shows that

$$
\max _{q_{n} \in S(Q)} \phi\left(q_{n}\right) \leq c-\varepsilon
$$


But since $Q \in \mathscr{A}_{k, i+1}$, we get that

$$
c=c_{k, i+1}=\inf _{F \in \mathscr{A}_{k, i+1}} \max _{q_{n} \in S(F)} \phi\left(q_{n}\right) \leq \max _{q_{n} \in S(Q)} \phi\left(q_{n}\right) \leq c-\varepsilon .
$$

This contradiction completes the proof of Lemma 5.32. Q.E.D.

Step 5. Proof of Proposition 5.1. By Lemmas 5.24 and 5.32, for any $(k, i) \geq$ $\left(k_{1}, 1\right), c_{k, i}$ is a positive critical value of $\phi$. Let $\mathscr{K}_{c_{k, i}}=\left(\phi^{\prime}\right)^{-1}(0) \cap \phi^{-1}\left(c_{k, i}\right)$. Then by Lemma 5.27,

$$
\mathscr{K}_{c_{k, i}} \subseteq W \backslash W_{0}, \quad \operatorname{dim} \mathscr{K}_{c_{k, i}}=1
$$

and $\mathscr{K}_{c_{k, i}}$ is an $S^{1}(W)$-invariant circle. Thus by Lemma 3.10 (for $\mathscr{W}$ )

$$
\gamma\left(\mathscr{K}_{c_{k, i}}\right)=1 \text { for }(k, i) \geq\left(k_{1}, 1\right) .
$$

Now (5.37) and $4^{\circ}$ of Lemma 5.32 show that

$$
0<c_{k_{1}, 1} \leq c_{k, i}<c_{k+1, i} \text { if }\left(k_{1}, 1\right) \leq(k, i) .
$$

Suppose for some $k_{2} \in \mathbf{N}, c_{k_{1}, 1}=\gamma_{k_{2}}$. Then combining with $1^{\circ}$ of Lemma 5.32 we get that

$$
c_{k, i} \geq c_{k, 1} \geq \gamma_{k_{2}+\left(k-k_{1}\right)}=\gamma_{k+m} \quad \forall(k, i) \geq\left(k_{1}, 1\right),
$$

where $m=k_{2}-k_{1}$. Therefore by Lemma 5.27, for $i=1, \ldots, N$,

$$
\frac{c_{k, i}}{k^{2}} \geq \frac{\gamma_{k+m}}{(k+m)^{2}} \cdot \frac{(k+m)^{2}}{k^{2}} \rightarrow+\infty \quad \text { as } k \rightarrow+\infty .
$$

Now the inequalities (5.8), $2^{\circ}$ of Lemma 5.24 and (5.38) imply (5.2). The proof is complete.

Remark 5.39. $1^{\circ}$. If in addition, $V$ satisfies the following condition (V3) There are $p>1$, and $a, b>0$ such that

$$
V(q) \leq a|q|^{p+1}+b \text { for } q \in \mathbf{R}^{N},
$$

then we can get more precise estimates for the growth rate of $\left\{a_{k, i}\right\}$. Since by Hölder's inequality

$$
V(q)+\frac{1}{2}|q|^{2} \leq(a+1) N^{(p-1) / 2} \sum_{n=1}^{N}\left|q_{n}\right|^{p+1}+(b+1) .
$$

Thus in the proof of Proposition 5.1, we may take

$$
G(s)=\alpha|s|^{p+1}, \quad g(s)=\alpha(p+1)|s|^{p-1} s \quad \text { for } s \in \mathbf{R}
$$

where $\alpha=(a+1) N^{(p-1) / 2}$. Then

$$
\phi(u)=\int_{0}^{2 \pi}\left(\frac{1}{2}|\dot{u}|^{2}-\alpha|u|^{p+1}\right) d t .
$$


By Remark 4.1 of [4], there is a constant $\beta>0$ such that the corresponding critical value

$$
\gamma_{k}=\beta k^{2(p+1) /(p-1)} \quad \text { for } k \in \mathbf{N} \text {. }
$$

So we get that there exists $m \in \mathbf{N}, \bar{\beta}>0$ such that for any $(k, i) \geq\left(k_{1}, 1\right)$

$$
\cdot a_{k, i} \geq \gamma_{k+m}-\bar{\beta} \geq \beta(k+m)^{2(p+1) /(p-1)}-\bar{\beta}
$$

i.e. there are constants $\alpha_{1}, \alpha_{2}>0$ such that

$$
a_{k, i} \geq \alpha_{1} k^{2(p+1) /(p-1)}-\alpha_{2} \quad \forall(k, i) \geq\left(k_{1}, 1\right) .
$$

$2^{\circ}$. For the functional

$$
\tilde{J}(q)=\int_{0}^{2 \pi}\left(\frac{1}{2}|\dot{q}|^{2}-U(t, q)\right) d t \quad \forall q \in E,
$$

if $U \in C\left(\mathbf{R} \times \mathbf{R}^{N}, \mathbf{R}\right)$ and is $2 \pi$-periodic in $t$, then the minimax value sequence $\left\{\tilde{a}_{k, i}\right\}$, which is defined by

$$
\tilde{a}_{k, i}{ }^{\prime}=\inf _{A \in \mathscr{A}_{k, i}} \max _{q \in A} \tilde{J}(q),
$$

possesses the same lower bound estimate for its growth rate as (5.2).

$3^{\circ}$. In their setting, Bahri and Berestycki obtained an estimate like (5.2) via Morse theory type arguments. See $\S 5$ of [4]. For us (5.2) is true for the whole sequence $\left\{a_{k, i}\right\}$, not only for one of its subsequences as in [4].

Finally, we can give the

Proof of Theorem 1.2. We assume $T=2 \pi$. A simple change of variables gives us the general case of $T$. Since $\mu>2$ implies $\mu /(\mu-1)<2,(5.2)$ and (4.6) imply that there exist infinitely many $(k, i) \in \mathscr{D}$ such that

$$
b_{k, i}>a_{k, i} \text { for }(k, i) \in \tilde{\mathscr{D}} \text {, }
$$

where $\tilde{\mathscr{D}}$ is the set of all such $(k, i)$ 's. Proposition 3.21 gives us an infinite sequence of critical values, $b_{k, i}(\delta)$ for $(k, i) \in \tilde{\mathscr{D}}$, of $J$, and

$$
b_{k, i}(\delta) \geq b_{k, i}>a_{k, i} \rightarrow+\infty \text { as } k \rightarrow+\infty \text { along } \tilde{\mathscr{D}} \text {. }
$$

By Lemma 2.8, there is $m \in \mathbf{N}$ such that for any $k \geq m,(k, i) \in \tilde{\mathscr{D}}, b_{k, i}(\delta)$ is also a critical value of $I$. Let $q_{k, i}$ be a critical point of $I$ corresponding to $b_{k, i}(\delta)$ for $k \geq m,(k, i) \in \tilde{\mathscr{D}}$. If $\left\{\left\|q_{k, i}\right\|_{L^{\infty}}\right\}$ were bounded, then the numbers

$$
\begin{aligned}
b_{k, i}(\delta) & =I\left(q_{k, i}\right)=\int_{0}^{2 \pi}\left(\frac{1}{2}\left|\dot{q}_{k, i}\right|^{2}-V\left(q_{k, i}\right)+f \cdot q_{k, i}\right) d t \\
& =\int_{0}^{2 \pi}\left(\frac{1}{2} q_{k, i} \cdot V^{\prime}\left(q_{k, i}\right)-V\left(q_{k, i}\right)+\frac{1}{2} f \cdot q_{k, i}\right) d t
\end{aligned}
$$

would also be bounded. Contrary to (5.41). Now the $q_{k, i}$ 's are $W^{1,2}\left(S^{1}, \mathbf{R}^{N}\right)$ solutions of (1.1). Since $f \in L^{2}\left(S^{1}, \mathbf{R}^{N}\right), q_{k, i} \in W^{2,2}\left(S^{1}, \mathbf{R}^{N}\right)$. The proof is complete. Q.E.D.

With the estimate (5.2), we can replace (V2) by a milder condition (V2') and still get the conclusion of Theorem 1.2. That is, 
Theorem 5.42. Let $V$ satisfy (V1) and the following condition,

$\left(\mathrm{V} 2^{\prime}\right)$

$$
\lim _{|q| \rightarrow+\infty} \frac{q \cdot V^{\prime}(q)}{|q|^{2}}=+\infty
$$

and there are $a, b>0$ such that

$$
\frac{1}{2} q \cdot V^{\prime}(q)-V(q) \geq a|q|^{2}-b \quad \forall q \in \mathbf{R}^{N} .
$$

Then the conclusion of Theorem 1.2 still holds.

We refer the readers to a related density result [12, Theorem 1.5], where we prove that (V1) and (5.43) implies that (1.1) is solvable if $f$ belongs to a certain dense subset of the space of all $T$-periodic functions in $L^{2}\left([0, T], \mathbf{R}^{N}\right)$.

\section{MORE GENERAL FORCED SYSTEMS}

Firstly we consider a more general non-autonomous Hamiltonian system

$$
\ddot{q}+U_{q}(t, q)=0
$$

where $U: \mathbf{R} \times \mathbf{R}^{N} \rightarrow \mathbf{R} . U_{q}$ is its gradient with respect to $q$. We have Theorem 6.2. Let $U$ satisfy the following conditions

(U1) $U \in C^{1}\left(\mathbf{R} \times \mathbf{R}^{N}, \mathbf{R}\right)$ and $U(t, q)$ is $T$-periodic in $t$ for some given $T>0$.

(U2) There exist $V: \mathbf{R}^{N} \rightarrow \mathbf{R}$ satisfying (V1), (V2) and constants $C>0$, $1 \leq \sigma \leq \mu / 2$ such that

$$
\left|U_{q}(t, q)-V_{q}(q)\right| \leq C\left(1+|q|^{\sigma-1}\right) \quad \text { for }(t, q) \in \mathbf{R} \times \mathbf{R}^{N} .
$$

Then (6.1) possesses infinitely many distinct $T$-periodic solutions.

To prove Theorem 6.2. We consider a functional

$$
I(q)=\int_{0}^{2 \pi}\left(\frac{1}{2}|\dot{q}|^{2}-V(q)+D(t, q)\right) d t
$$

where $D(t, q)=V(q)-U(t, q)$. In $\S \S 3$ and 5 replacing $f \cdot q$ by $D(t, q)$, we can go through the proofs and get

$$
a_{k, i} \leq \beta k^{\mu /(\mu-\sigma)} \quad \forall k \geq k_{1}, 1 \leq i \leq N .
$$

(6.4), (5.2), and $2^{\circ}$ of Remark 5.42 show that to get Theorem 6.2 we need $\mu /(\mu-\sigma) \leq 2$, i.e. $\sigma \leq \mu / 2$.

Similarly we have 
Theorem 6.5. If in addition $V$ satisfies (V3) in Remark 5.42, then the conclusion of Theorem 6.2 holds with $\sigma<\mu(p+3) /(2(p+1)),(\sigma<(\mu+2) / 2$, if $p+1=\mu)$.

Next we consider a forced Lagrangian system

$$
\frac{d}{d t} \frac{\partial L}{\partial p}(q, \dot{q})-\frac{\partial L}{\partial q}(q, \dot{q})=f(t)
$$

where the Lagrangian function $L$ is given by

$$
L(q, p)=\sum_{i, j=1}^{N} a_{i j}(q) p_{i} p_{j}+\sum_{i=1}^{N} b_{i}(q) p_{i}-V(q) \quad \forall(q, p) \in \mathbf{R}^{N} \times \mathbf{R}^{N} .
$$

We assume the following conditions on $L$,

(L1) $a_{i j}, b_{i}, V \in C^{1}\left(\mathbf{R}^{N}, \mathbf{R}\right)$ and $a_{i j}=a_{j i}$, for $i, j=1, \ldots, N$.

(L2) There are constants $\mu>2, \bar{r}>0$ such that

$$
0<\mu V(q) \leq q \cdot V^{\prime}(q) \quad \forall|q| \geq \bar{r} .
$$

(L3) There are constants $\lambda>0, \tau \in(0, \mu-2)$ such that

$$
\sum_{i, j=1}^{N} a_{i j}(q) p_{i} p_{j} \geq \lambda|p|^{2} \quad \forall(q, p) \in \mathbf{R}^{N} \times \mathbf{R}^{N},
$$

and

$$
\tau \sum_{i, j=1}^{N} a_{i j}(q) p_{i} p_{j} \geq \sum_{i, j=1}^{N}\left(q \cdot a_{i j}^{\prime}(q)\right) p_{i} p_{j} \quad \forall(q, p) \in \mathbf{R}^{N} \times \mathbf{R}^{N} .
$$

$$
\lim _{|q| \rightarrow+\infty} \frac{|b(q)|^{2}}{V(q)}=0 \quad \text { and } \quad \lim _{|q| \rightarrow+\infty} \frac{\left|b^{\prime}(q) q\right|^{2}}{q \cdot V^{\prime}(q)}=0 .
$$

Assume $f(t)$ is $T$-periodic in $t$ and $f \in L^{2}\left([0, T], \mathbf{R}^{N}\right)$. Then we have the following

Theorem 6.7. Under the above conditions, for any $R>0,(6.6)$ possesses $a$ solution $q \in W^{2,2}\left([0, T], \mathbf{R}^{N}\right)$ with period $T$ such that $\|q\|_{L^{\infty}} \geq R$.

We omit the proofs of above theorems.

Remark 6.8. $1^{\circ}$. Theorem 6.2 gives a result different from Bahri and Berestycki's Theorem 6 [4].

$2^{\circ}$. Greco gave a result for $(6.6)$ [11, Theorem 1.1]. In addition to $(\mathrm{L} 1)-(\mathrm{L} 3)$ he assumed (V3) and that $|b(q)|,\left|b^{\prime}(q) q\right|$ were bounded.

$3^{\circ}$. We also refer to Benci, Cappozzi, and Fortunato [5] for a related result.

APPENDIX. A DETAILED STUdY OF A SINGLE EQUATION

In this appendix, we give a direct proof of Lemma 5.27 for the equation

$$
\ddot{v}+g(v)=0
$$


corresponding to the functional $\phi$ defined by (5.4). We are indebted to Professor Paul H. Rabinowitz who suggested this argument.

In the proof of Lemma 5.3 we further require that $a_{n+1} \geq 50 n^{2} \sum_{i=1}^{n} a_{i}, \forall n \in$ $\mathbf{N}$. Then $G$ and $g$ satisfy the following conditions:

(A.2) $G \in C^{2}(\mathbf{R}, \mathbf{R}), G^{\prime}=q$ is odd and $G(0)=g(0)=g^{\prime}(0)=0$.

(A3) There is a constant $\mu>2$ such that $0<\mu G(r) \leq g(r) r$ for $r \neq 0$.

(A.4) $2 G(r) g^{\prime}(r)-g^{2}(r)>0$ for $r>0$.

(A.5) $G(r) /\left(r^{2}\right) \rightarrow 0$ as $r \rightarrow 0$.

Conditions (A.2), (A.3) and (A.4) imply that $g(r)>0, g^{\prime}(r)>0$ and $\frac{d}{d r}(g(r) / r)$

$>0$ for $r>0$, and that $G$ is even.

Define $F$ by

$$
F=\frac{1}{2} y^{2}+G(x) .
$$

The simple closed curves $F=$ constants are the trajectories of (A.1) in the phase plane so all solutions of (A.1) are periodic and the periods satisfy

$$
T(F)=\frac{d A(F)}{d F}
$$

where $A(F)$ is the area of the region enclosed by the curve for fixed $F$. It is easy to see that all $T$-periodic solutions of $(\mathrm{A} .1)$ in $W^{1,2}([0, T], \mathbf{R})$ are $C^{2}$. Note that for any given value of $F$, by (A.6)

$$
y= \pm \sqrt{2(F-G(x))},
$$

so since $G$ is even in $x$, the solutions of (A.1) spend equal amounts of time in each quadrant in the phase plane and the solution $v$ of (A.1) with the energy $F$, period $T=T(F)$ and initial value $v(0)=0$ is odd about 0 and even about $T / 4$. From (A.6) we also have that $v(t)=0$ only at $t=i T / 2$ for $i \in \mathbf{Z}$ and for such $t,|\dot{v}(t)|=\sqrt{2 F}$.

Since

$$
A(F)=4 \int_{0}^{x_{0}(F)} y(F, x) d x
$$

where $G\left(x_{0}(F)\right)=F$ and $y\left(F, x_{0}(F)\right)=0$, from (A.7) we get that

$$
\begin{aligned}
T(F) & =4 y\left(F, x_{0}(F)\right) \frac{d x_{0}(F)}{d F}+4 \int_{0}^{x_{0}(F)} \frac{\partial y}{\partial F}(F, x) d x \\
& =4 \int_{0}^{G^{-1}(F)}\{2(F-G(x))\}^{-1 / 2} d x .
\end{aligned}
$$

Remark A.9. For any constant $F>0, t=\int_{0}^{v}\{2(F-G(x))\}^{-1 / 2} d x$ defines a function $t$ of $v$ on $\left[0, G^{-1}(F)\right]$. Since $d t / d v=\{2(F-G(v))\}^{-1 / 2} \neq 0$ for $v \in\left(0, G^{-1}(F)\right)$, by the implicit function theorem, we get its inverse function $v=v(t)$ defined on $(0, T / 4)$ with $d v / d t=\sqrt{2(F-G(v))}$, where $T=T(F)$ is 
defined by (A.8). Define $v(0)=0, v(T / 4)=G^{-1}(F), \dot{v}(0)=\sqrt{2 F}, \dot{v}(T / 4)=$ 0 and define $v(t)=v(T / 2-t)$ for $T / 4<t \leq T / 2, v(t)=-v(T-t)$ for $T / 2<$ $t \leq T$. Extend $v \quad T$-periodically to $\mathbf{R}$. Then we have that $v \in C^{1}(\mathbf{R}, \mathbf{R})$, is $T$-periodic, odd about 0 , even about $T / 4$ and is a weak solution of (A.1), i.e. for every $T$-periodic $\varphi \in C^{\infty}(\mathbf{R}, \mathbf{R})$,

$$
\int_{0}^{T} \dot{v} \cdot \dot{\varphi} d t=\int_{0}^{T} g(v) \varphi d t
$$

It then easily follows that $v \in C^{2}(\mathbf{R}, \mathbf{R})$, has the energy $F$ and minimal period $T(F)$. We denote this solution by $v_{F}$.

Lemma A.10. $1^{\circ}$. T is a strictly monotone decreasing continuous function of $F$ on $(0,+\infty)$.

$$
\begin{aligned}
& 2^{\circ} . T \rightarrow 0 \text { as } F \rightarrow+\infty . \\
& 3^{\circ} . T \rightarrow+\infty \text { as } F \rightarrow 0 .
\end{aligned}
$$

Proof. $1^{\circ}$. The continuity of $T$ is a direct consequence of (A.8).

Assume that there are $F_{1}<F_{2}$ such that $T_{1}=T\left(F_{1}\right) \leq T\left(F_{2}\right)=T_{2}$. For $i=1,2$ let $v_{i}=v_{F_{i}}$ be the solution of (A.1) defined in (A.9) with the energy $F_{i}$. Then $v_{i}$ has period $T_{i}$ and $v_{i}(t)=0$ at $t=0, T_{i} / 2$ and $T_{i}, v_{i}(t)>0$ on $\left(0, T_{i} / 2\right), v_{i}(t)<0$ on $\left(T_{i} / 2, T_{i}\right) . \dot{v}_{1}(0)=\sqrt{2 F_{1}}<\sqrt{2 F_{2}}=\dot{v}_{2}(0)$, since $v_{i}$ satisfies

$$
\ddot{v}+\psi(v) v=0,
$$

where $\psi(v)=g(v) / v$ satisfies $\psi(0)=0$ by (A.2) and is strictly monotone increasing since $d / d r(g(r) / r)>0$ for $r>0$. Hence by Sturm Comparison Theorem (cf. Theorem 1.1, Chapter 8 of [7]), it is impossible that $v_{2}(t)>v_{1}(t)$ for every $t \in\left(0, T_{1} / 2\right)$, i.e. there is a first $\xi \in\left(0, T_{1} / 2\right)$ such that $v_{1}(\xi)=$ $v_{2}(\xi)$. By (A.6), $\left|\dot{v}_{2}(\xi)\right|>\left|\dot{v}_{1}(\xi)\right|$. If $\xi>T_{1} / 4, v_{2}(t)>v_{1}(t)$ on $\left(0, T_{1} / 4\right]$. Then by the symmetricities about $T_{1} / 4$ and $T_{2} / 4$ separately, $v_{2}(t)>v_{1}(t)$ on $\left(0, T_{1} / 2\right)$ contrary to $\xi \in\left(0, T_{1} / 2\right)$. If $\xi \leq T_{1} / 4$, since $v_{i}$ is even about $T_{i} / 4$ and is convex on $\left(0, T_{i} / 2\right)$ by $(\mathrm{A} .1)$, we have $\dot{v}_{2}(\xi)>\dot{v}_{1}(\xi)$. Therefore $v_{2}(t)<v_{1}(t)$ for $t<\xi$ and near $\xi$. This contradicts the definition of $\xi$. Thus $T$ must be a strictly monotone decreasing function of $F$. 
$2^{\circ}$. In (A.8), let $s=G(x)$, then

$$
\begin{aligned}
T & =2 \sqrt{2}\left(\int_{0}^{F / 2}+\int_{F / 2}^{F} \frac{1}{\sqrt{F-s} g\left(G^{-1}(s)\right)} d s\right) \\
& \leq \frac{4}{\sqrt{F}} \int_{0}^{F / 2} \frac{d}{d s}\left(G^{-1}(s)\right) d s+\frac{2 \sqrt{2}}{g\left(G^{-1}\left(\frac{F}{2}\right)\right)} \int_{F / 2}^{F} \frac{1}{\sqrt{F-s}} d s \\
& =\frac{4}{\sqrt{F}} G^{-1}\left(\frac{F}{2}\right)+4 \sqrt{2} \frac{\sqrt{F / 2}}{g\left(G^{-1}(F / 2)\right)} \\
& \leq 4 \frac{G^{-1}(F / 2)}{\sqrt{F}}+4 \sqrt{2} \frac{\sqrt{F / 2} G^{-1}(F / 2)}{\mu G\left(G^{-1}(F / 2)\right)} \quad \text { is strictly increasing) } \\
& \leq 8 \frac{G^{-1}(F / 2)}{\sqrt{F / 2}} .
\end{aligned}
$$

By (A.3), there are constants $\alpha, \beta>0$ such that $G(r) \geq \alpha r^{\mu}-\beta$ for $r \geq 0$. So $r \leq((G(r)+\beta) / \alpha)^{1 / \mu}$. Let $r=G^{-1}(F / 2)$, then $G^{-1}(F / 2) \leq((F / 2+\beta) / \alpha)^{1 / \mu}$. Thus

$$
T \leq 8\left(\frac{1}{\alpha}\left(\frac{F}{2}+\beta\right)\right)^{1 / \mu}\left(\frac{F}{2}\right)^{-1 / 2} \rightarrow 0 \text { as } F \rightarrow+\infty .
$$

$3^{\circ}$. In (A.5) let $r=G^{-1}(F)$ then $\sqrt{F} / G^{-1}(F) \rightarrow 0$ as $F \rightarrow 0$ and from (A.8)

$$
T \geq 4 \int_{0}^{G^{-1}(F)}(2 F)^{-1 / 2} d x=4 \sqrt{2} \frac{G^{-1}(F)}{\sqrt{F}} \rightarrow+\infty \quad \text { as } F \rightarrow 0 .
$$

This completes the proof. Q.E.D.

Therefore for any given $T>0$ there is a unique $F(T)$ which gives a solution $v$ of (A.1) with the energy $F(T)$ and period $T$.

Now we consider all $2 \pi$-periodic solutions of (A.1). Let $F_{k}$ be the energy level corresponding to the period $2 \pi / k$. Let $u_{k}=v_{F_{k}}$ defined in Remark A.9 and let

$$
\gamma_{k}=\phi\left(u_{k}\right) \equiv \int_{0}^{2 \pi}\left(\frac{1}{2}\left|\dot{u}_{k}\right|^{2}-G\left(u_{k}\right)\right) d t \quad \text { for } k \in \mathbf{N}
$$

\section{Lemma A.12.}

$$
\frac{F_{k}}{k^{2}} \geq \frac{4}{\pi^{2}} G^{-1}\left(F_{k}\right) \quad \text { for } k \in \mathbf{N}
$$

Proof. By (A.11), $2 \pi / k \geq 4 G^{-1}\left(F_{k}\right)\left(2 F_{k}\right)^{-1 / 2}$ and this implies the lemma. Q.E.D.

Lemma A.13.

$$
\frac{\gamma_{k}}{k^{2}} \geq \frac{4(\mu-2)}{\pi \mu} G^{-1}\left(F_{k}\right) \text { and } \gamma_{k}>0 \text { for } k \in \mathbf{N}
$$


Proof. Since $\int_{0}^{2 \pi}\left|\dot{u}_{k}\right|^{2} d t=\int_{0}^{2 \pi} g\left(u_{k}\right) u_{k} d t$ and by (A.3), we have that

$$
\begin{aligned}
\gamma_{k} & =\int_{0}^{2 \pi}\left\{\left(\frac{1}{2}-\frac{1}{\mu}\right)\left|\dot{u}_{k}\right|^{2}+\left(\frac{1}{\mu} g\left(u_{k}\right) u_{k}-G\left(u_{k}\right)\right)\right\} d t \\
& \geq\left(\frac{1}{2}-\frac{1}{\mu}\right) \int_{0}^{2 \pi}\left(\frac{1}{2}\left|\dot{u}_{k}\right|^{2}+\frac{1}{2} u_{k} g\left(u_{k}\right)\right) d t \\
& \geq\left(\frac{1}{2}-\frac{1}{\mu}\right) \int_{0}^{2 \pi}\left(\frac{1}{2}\left|\dot{u}_{k}\right|^{2}+G\left(u_{k}\right)\right) d t \\
& =\frac{\pi(\mu-2)}{\mu} F_{k}>0 .
\end{aligned}
$$

Combining with Lemma A.12 we complete the proof. Q.E.D.

Lemmas A.10 and A.13 yield

$$
\lim _{k \rightarrow+\infty} \frac{\gamma_{k}}{k^{2}} \geq \frac{4(\mu-2)}{\pi \mu} \lim _{k \rightarrow+\infty} G^{-1}\left(F_{k}\right)=+\infty
$$

Lemma A.15. $\gamma_{k}<\gamma_{k+1}$ for $k \in \mathbf{N}$.

Proof. We have that $\int_{0}^{2 \pi}\left|\dot{u}_{k}\right|^{2} d t=\int_{0}^{2 \pi} g\left(u_{k}\right) u_{k} d t, u_{k}$ has minimal period $2 \pi / k$, is odd about 0 and even about $\pi /(2 k)$, is positive and strictly increasing on $(0, \pi /(2 k)]$. Therefore

$$
\gamma_{k}=\int_{0}^{2 \pi}\left(\frac{1}{2} g\left(u_{k}\right) u_{k}-G\left(u_{k}\right)\right) d t=4 k \int_{0}^{\pi /(2 k)}\left(\frac{1}{2} g\left(u_{k}\right) u_{k}-G\left(u_{k}\right)\right) d t .
$$

Let

$$
\left.y=\sqrt{2\left[F_{k}-G\left(u_{k}(t)\right)\right.}\right] \text { for } t \in[0, \pi /(2 k)]
$$

and $h(x)=(g(x) x / 2-G(x)) / g(x)$ for $x \geq 0$, then

$$
\frac{\gamma_{k}}{4 k}=\int_{0}^{\sqrt{2 F_{k}}} h \circ G^{-1}\left(F_{k}-\frac{1}{2} y^{2}\right) d y \text {. }
$$

Since $h^{\prime}(x)=\left(2 G(x) g^{\prime}(x)-g^{2}(x)\right) /\left(2 g^{2}(x)\right)>0$ and $(d / d x) G^{-1}(x)=$ $1 / g\left(G^{-1}(x)\right)>0$ for $x>0 . h, G^{-1}$ and $h \circ G^{-1}$ are strictly increasing on $[0,+\infty)$.

By Lemma A.10, $F_{k+1}>F_{k}$. So for $s \in\left[0, \sqrt{2 F_{k}}\right]$

$$
F_{k+1}-\frac{1}{2}\left(\sqrt{2 F_{k+1}}-s\right)^{2} \geq F_{k}-\frac{1}{2}\left(\sqrt{2 F_{k}}-s\right)^{2} .
$$

Therefore for $k \in \mathbf{N}$

$$
\begin{aligned}
\frac{\gamma_{k+1}}{4(k+1)}= & \int_{\sqrt{2 F_{k+1}}-\sqrt{2 F_{k}}}^{\sqrt{2 F_{k+1}}}+\int_{0}^{\sqrt{2 F_{k+1}}-\sqrt{2 F_{k}}}\left\{h \circ G^{-1}\left(F_{k+1}-\frac{1}{2} y^{2}\right)\right\} d y \\
\geq & \int_{0}^{\sqrt{2 F_{k}}}\left\{h \circ G^{-1}\left(F_{k}-\frac{1}{2} y^{2}\right)\right\} d y \\
& +\int_{0}^{\sqrt{2 F_{k+1}}-\sqrt{2 F_{k}}}\left\{h \circ G^{-1}\left(F_{k+1}-\frac{1}{2} y^{2}\right)\right\} d y>\frac{\gamma_{k}}{4 k} .
\end{aligned}
$$


This yields that $\gamma_{k}<\gamma_{k+1}$ for $k \in \mathbf{N}$ and completes the proof. Q.E.D.

For $k \in \mathbf{N}$, we define $\mathscr{K}_{\gamma_{k}}=\left(\phi^{\prime}\right)^{-1}(0) \cap \phi^{-1}\left(\gamma_{k}\right)$.

Lemma A.16. For $k \in \mathbf{N}, \mathscr{K}_{\gamma_{k}}=\left\{T_{\theta} u_{k} \mid \theta \in[0,2 \pi]\right\}$.

Proof. If $v \not \equiv 0$ is a $2 \pi$-periodic solution of (A.1), then there is $k \in \mathbf{N}$ such that $v$ has minimal period $2 \pi / k$. We claim that there exists at least a $\theta \in$ $[0,2 \pi / k]$ such that $v(\theta)=0$. For otherwise, integrating (A.1) from 0 to $2 \pi / k$ we get $\int_{0}^{2 \pi} g(v) d t=0$. This is a contradiction. Let $u=T_{\theta} v$, then $u(0)=0, u$ has minimal period $2 \pi / k$, by Lemma A.10 $u$ has the energy $F_{k}$. From (A.6) $u$ is odd about 0 and even about $\pi /(2 k)$, on $[0, \pi /(2 k)] u$ satisfies either $d u / d t=\sqrt{2\left(F_{k}-G(u)\right)}$ or $d u / d t=-\sqrt{2\left(F_{k}-G(u)\right)}$. Therefore either $u=u_{k}$ or $u=-u_{k}$. That is either $v=T_{-\theta} u_{k}$ or $v=T_{\pi / k-\theta} u_{k}$. Since $\phi$ is $S^{1}$-invariant, combining with Lemma A.15 the proof is complete. Q.E.D.

Now Remark A.9, (A.14), Lemmas A.15 and A.16 give us the conclusion of Lemma 5.27.

\section{REFERENCES}

1. R. A. Adams, Sobolev spaces, Academic Press, New York, 1975.

2. A. Bahri and H. Berestycki, A perturbation method in critical point theory and applications, Trans. Amer. Math. Soc. 167 (1981), 1-32.

3. __ Forced vibrations of superquadratic Hamiltonian systems, Acta Math. 152 (1984), 143197.

4. __ Existence of forced oscillations for some nonlinear differential equations, Comm. Pure Appl. Malth. 37 (1984), 403-442.

5. V. Benci, A. Cappozi and D. Fortunato, Periodic solutions of Hamiltonian systems of prescribed period, Mathematics Research Center, Technical Summary Report No. 2508, Univ. of Wisconsin-Madison, 1983.

6. H. Berestycki, Solutions périodiques de systémes hamiltoniens, Séminaire Bourbaki, 35e année, 1982/83, No. 603, pp. 105-128.

7. E. A. Coddington and N. Levinson, Theory of ordinary differential equations, McGraw-Hill, New York, 1955.

8. J. Dugundji, An extension of Tietze's theorem, Pacific J. Math. 1 (1951), 353-367.

9. E. R. Fadell, S. Husseini and P. H. Rabinowitz, Borsuk-Ulam theorems for arbitrary $S^{1}$-actions and applications, Trans. Amer. Math. Soc. 174 (1982), 345-360.

10. E. R. Fadell and P. H. Rabinowitz, Generalized cohomological index theories for Lie group actions with an application to bifurcation questions for Hamiltonian system, Invent. Math. 45 (1978), 139-174.

11. C. Greco, On forced oscillations of Lagrangian systems, Preprint 1986.

12. Y. Long, On the density of the range for some superquadratic operators, Mathematics Research Center, Technical Summary Report No. 2859, Univ. of Wisconsin-Madison, 1985.

13. __ Multiple solutions of perturbed superquadratic second order Hamiltonian systems, Mathematics Research Center, Technical Summary Report No. 2963, Univ. of Wisconsin-Madison, 1987.

14. P. H. Rabinowitz, Variational methods for nonlinear eigenvalue problems, Eigenvalues of Nonlinear Problems, Ediz. Cremonese, Roma, 1974.

15. __ Periodic solutions of Hamiltonian systems, Comm. Pure Appl. Math. 31 (1978), 157-184. 
16. __ On large norm periodic solutions of some differential equations, Ergodic Theory and Dynamical Systems. II (A. Katok, Ed., College Park, Md., 1979-1980), Birkhäuser, 1982.

17. _ Periodic solutions of Hamiltonian systems: A survey, SIAM J. Math. Anal. 13 (1982), 343-352.

18. _ Multiple critical points of perturbed symmetric functionals, Trans. Amer. Math. Soc. 272 (1982), 753-769.

19. _ Periodic solutions of large norm of Hamiltonian systems, J. Differential Equations 50 (1983), 33-48.

20. _ Minimax methods in critical point theory with applications to differential equations, CBMS Regional Conf. Ser. in Math., no. 65, Amer. Math. Soc., Providence, R.I., 1986.

Department of Mathematics, University of Wisconsin-Madison, Madison, Wisconsin 53706

Current address: Nankai Institute of Mathematics, Nankai University, Tianjin, The People's Republic of China 